\title{
Nonlinear dynamical analysis of GNSS data: quantification, precursors and synchronisation
}

\author{
Bruce Hobbs ${ }^{1,3^{*}}$ (D) and Alison Ord ${ }^{1,2}$
}

\begin{abstract}
The goal of any nonlinear dynamical analysis of a data series is to extract features of the dynamics of the underlying physical and chemical processes that produce that spatial pattern or time series; a by-product is to characterise the signal in terms of quantitative measures. In this paper, we briefly review the methodology involved in nonlinear analysis and explore time series for GNSS crustal displacements with a view to constraining the dynamics of the underlying tectonic processes responsible for the kinematics. We use recurrence plots and their quantification to extract the invariant measures of the tectonic system including the embedding dimension, the maximum Lyapunov exponent and the entropy and characterise the system using recurrence quantification analysis (RQA). These measures are used to develop a data model for some GNSS data sets in New Zealand. The resulting dynamical model is tested using nonlinear prediction algorithms. The behaviours of some RQA measures are shown to act as precursors to major jumps in crustal displacement rate. We explore synchronisation using cross- and joint-recurrence analyses between stations and show that generalised synchronisation occurs between GNSS time series separated by up to $600 \mathrm{~km}$. Synchronisation between stations begins up to 250 to 400 days before a large displacement event and decreases immediately before the event. The results are used to speculate on the coupled processes that may be responsible for the tectonics of the observed crustal deformations and that are compatible with the results of nonlinear analysis. The overall aim is to place constraints on the nature of the global attractor that describes plate motions on the Earth.
\end{abstract}

Keywords: GNSS time series, Nonlinear analysis, Dynamical systems, Recurrence plots, Recurrence quantification analysis (RQA), Cross and joint recurrence plots, Crustal deformation, Precursors, Synchronisation

\section{Introduction}

The general nature of the dynamics of the mantle of the Earth along with the interaction of the mantle with the lithosphere is thought to be well known; broadly, convective motion in the mantle with coupled thermal and mass transport results in tractions on the bases of the lithospheric plates. These tractions together with other tractions generated by instabilities, such as subducting slabs along with forces generated by spreading from mid-ocean ridges, lead to plate motions expressed as plate deformations observed at the surface of the Earth in the form of GNSS (Global Navigation Satellite

\footnotetext{
* Correspondence: bruce.hobbs@csiro.au

${ }^{1}$ Centre for Exploration Targeting, School of Earth Sciences, University of Western Australia, Perth, Western Australia 6009, Australia

${ }^{3}$ CSIRO, Perth, Western Australia 6102, Australia

Full list of author information is available at the end of the article
}

System) measurements. Fundamental questions are do the displacements we observe synchronise in some way from one place to another? And if so, on what spatial and time scales does synchronisation occur? Can the pattern of synchronisation be used to define precursors to major and commonly destructive displacement events? The global array of GNSS measurements and their time series should, on principle, give enough information to construct the dynamics of the underlying processes and answer such questions. However, in order to be more specific, one needs to better express the partial differential equations that describe the processes responsible for the dynamics and the ways in which these processes are coupled and evolve with time. With the present uncertainties regarding constitutive relations and properties and the temperature distribution within the Earth, it is difficult to constrain possible geometric and kinematic 
models for plate development and evolution with computer models based on current knowledge of these issues and with the available computing power. The results of any such models should at least be compatible with the results of detailed analysis of observed crustal displacement data which is the purpose of this paper.

The main data sets we have at present that are useful in developing such constraints are geophysical data sets (gravity, magnetics and seismic), heat flow measurements, the distribution of topography on the surface of the Earth and GNSS data on crustal displacements. These latter data sets are now well distributed over the Earth, and in some instances, continuous time series go back at least a decade. We concentrate on GNSS data in this paper with the aim of establishing how much of the dynamics of the plate tectonic processes is reflected in such data. Future papers attempt to integrate these data sets. Just as the global weather system is an expression of the Navier-Stokes equations for a viscous fluid with coupled heat and mass transport and which result in highly nonlinear behaviour, we expect the dynamics underlying plate tectonics to be highly nonlinear. The aim is to characterise and quantify this behaviour and, as far as is possible, move towards identifying the mathematical expression of the coupled processes that operate to produce crustal deformation driven by plate motions.

\section{Nonlinear time series analysis and dynamical systems}

\section{The nature of nonlinear time series analysis}

The aim of time series analysis is to classify and quantify the nature of a particular time series of interest and, if possible, understand the dynamics of the processes that operated to produce the time series. Most approaches to such a task in the geosciences consider linear stochastic models commonly with an assumed Gaussian or log-normal distribution for the data. This essentially means that one assumes the data to be stochastic, that is, the result of uncorrelated linear processes. The linear assumption implies that the law of superposition (Hobbs and Ord 2015, pp. 10-15) is valid so that if $f(x)$ and $g(x)$ describe the dynamics of a system, then linear combinations of $f(x)$ and $g(x)$ also define the dynamics. Such an assumption implies that Fourier methods are useful in characterising the data (Stoica and Moss 2005). The stochastic assumption assumes no long-range correlations in the data. Analysis is difficult if the data are non-stationary (the mean and/or the standard deviation vary with time), there is considerable noise in the data and "outliers" (departures from Gaussian or log-normal distributions) are common. Nevertheless, the data are often forced to fit stationary, Gaussian distributions with no long-range correlations and methods such as kriging, co-kriging, autoregressive and moving average methods and power spectra together with filtering/smoothing procedures are employed. Such methods are parametric (a statistical distribution is assumed for the data) and have no conceptual link to the underlying processes that produced the data. The stationary stochastic Gaussian time series, consisting of the terms $\left\{x_{1}, x_{2}, \ldots . ., x_{N}\right\}$, where $\mathrm{N}$ is the total number of terms, is commonly characterised using Fourier transform methods and by the autocorrelation function, $c(\tau)$ :

$$
c(\tau)=\frac{\left\langle\left(x_{i}-x_{i+\tau}\right)^{2}\right\rangle}{\left\langle x_{i}^{2}\right\rangle}
$$

where $\tau$ is called the lag and the $\langle "\rangle$ brackets denote the mean of the quantities involved (Box et al. 2008). Noise reduction is commonly thought of as a smoothing operation, the premise being that smooth data are better in some unspecified way than irregular data, and is commonly undertaken using recursive Bayesian procedures such as in the Kalman filter and its variants (Judd and Stemler 2009).

The outcome of any time or spatial series analysis is a data model which enables one to characterise the statistical measures (mean, standard deviation, autocorrelation function, power spectrum and so on) of the data and if possible undertake forecasts, interpolations and extrapolations of the data. We distinguish two classes of data models; one is a parametric stochastic data model that assumes an underlying statistical distribution and has no relation to the underlying processes that produced the data. The other is a non-parametric deterministic data model that makes no assumptions about the underlying statistics and directly reflects the dynamics of the system. The linear, stochastic procedures of kriging, co-kriging, autoregressive and moving average methods work well for linear systems where the law of superposition holds and Fourier methods clearly delineate discrete periodicities in the data. These are methods of constructing a stochastic, parametric data model. However, in nonlinear systems, especially those that are chaotic, these methods fail; the assumptions of Gaussian or log-normal distributions with no long-range correlations break down. Nonlinear signal processing methods (Small 2005) become not only essential but are capable of delineating the nature of the processes that operated or of testing models of processes that might be proposed (Judd and Stemler 2009; Small 2005). We paraphrase Judd and Stemler (2010): Understanding: it is not about the statistics, it is about the dynamics.

Part of the reason why linear parametric procedures fail for nonlinear systems that arise from a number of coupled processes is that in nonlinear systems the data for a particular quantity are a projection of processes from a higher dimensional state space on to that single 
quantity. Thus, for a sliding frictional surface where the only processes might be velocity-dependent frictional softening, accompanied by heat production and chemical healing of damage, the behaviour is described in a four-dimensional state space with coordinates comprising the state variables, velocity, temperature, friction coefficient and degree of chemical healing. A time series for temperature is a projection from the four-dimensional state space on to a one-dimensional time series. Quantities that appear close together in the time series may in fact be widely separated in state space. With respect to the GNSS time series from New Zealand that we examine in this paper, the deforming crustal system operates in a state space where at least the state variables velocity, stress, strain-rate, temperature, damage-rate, healing-rate and fluid pressure are needed to define the system; there probably are others involving the ways in which one part of the system is coupled to other parts. The GNSS displacement signal we observe is the projection from a space defined by these state variables on to a single displacement record that we observe at a particular station as a one dimensional time series.

As opposed to stochastic data models based on Gaussian statistics, lack of long-range correlations and the principle of superposition, the nonlinear systems we are interested in studying in the geosciences result from clearly defined physical and chemical processes. Although we may have considerable trouble in discovering and characterising these processes, the system is deterministic rather than stochastic. Hence, in principle, we should be able to define for a system of interest the invariant measures that characterise the system. An invariant measure remains the same independently of the way in which the system is observed and so remains the same independently of the dimensions of the state space in which we observe the system. Such measures include the Rényi generalised dimensions (including the fractal support dimension and the correlation dimension for the system) that characterise the geometry and are defined from a multifractal spectrum for the system (Beck and Schlögl 1995; Arneodo et al. 1995; Ord et al., 2016), the Lyapunov exponents that are related to the dynamics of the system and define the stability of the system and how far prediction is possible (Small 2005) and the Kolmogorov-Sinai entropy, related to information theory, that tells us how much information exists in the signal and is also related to predictability (Beck and Schlögl 1995; Small 2005). We will estimate these invariant measures for GNSS time series together with a number of other quantitative measures but not dwell too heavily on the mathematics behind the theory. Readers who require in-depth treatments should consult (Abarbanel 1996; Beck and Schlögl 1995; Sprott 2003; Kantz and Schreiber 2004; Small 2005; Judd and Stemler 2010). This paper is a brief review of nonlinear analysis with an emphasis on recurrence methods (Marwan et al. 2007a). The principles are illustrated using specific examples from the Lorentz system (Sprott 2003, p. 205) and several GNSS time series from New Zealand.

\section{The invariant measures}

The basis of nonlinear analysis lies in powerful proposals put forward by Crutchfield (1979) and Packard et al. (1980) and proved rigorously by Takens (1981). What is commonly referred to as Takens' theorem states that the complete dynamics of a system can be derived from a time series for a single state variable from that system. The reason for this (as expressed in the friction example above) is that in systems where all the state variables are coupled, the behaviour of one depends on the behaviours of all the others and so the time series for one variable has the behaviours of all the other variables encoded within it. Thus, if we have a time series $\left\{x_{1}, x_{2}, \ldots . ., x_{N}\right\}$, then we can construct $\mathbb{M}$-dimensional reconstruction space vectors, $\boldsymbol{M}(t)$, from $\mathbb{M}$ time delayed samples so that the vector $\boldsymbol{M}$ is:

$$
\boldsymbol{M}(t)=[x(t), x(t+\tau), x(t+2 \tau), \ldots \ldots \ldots, x(t+(\mathbb{M}-1) \tau)]
$$

In this process, every point in the signal is compared with a point distant $\tau$ away. These vectors define the attractor for the system; this is the manifold that all possible states of the system can occupy independently of the initial conditions. If in this construction, the delay, $\tau$, is small, the coordinates comprising $\boldsymbol{M}$ are strongly correlated and so the reconstructed attractor lies close to the diagonal of the reconstruction space. It is something of an art form to select $\tau$ such that the dynamics unfold off that diagonal. The attractor describing the complete dynamics of the system is embedded in a space which has a dimension that reflects the number of state variables in the system dynamics. The state space in which the attractor "lives" has dimensions, $\mathbb{D}$. If $\mathbb{M}$ exceeds $\mathbb{D}$, the attractor does not change and $\mathbb{D}$ is called the embedding dimension. For very large dimension systems, it may prove very difficult to construct an attractor that looks interesting or meaningful. This is simply because we are projecting a $\mathbb{D}$-dimensional object into two or three dimensions. If we explore the system in a space that has dimensions less than $\mathbb{D}$ then evolutionary trajectories of the system appear to cross one another because of the problems in projecting the trajectories from a higher dimensional space. Points on trajectories that appear close in the observational space but in fact are far apart in $\mathbb{D}$-space are called false neighbours. Algorithms for calculating the number of false neighbours in a given data set are given by Sprott (2003) and Small (2005). If 
one can identify a dimension where the number of false neighbours is zero then one has a good estimate of the embedding dimension. For white noise, the percentage of false neighbours remains at $50 \%$ independent of the dimension of the space in which the signal is observed.

One can also identify another dimension that we call the dynamical or state dimension, $D$. This is the dimension, it may or may not be an integer, that is the true dimension of the attractor. Generally, $D$ is difficult to measure because of noise and $\mathbb{D}$ is attenuated because of non-stationary behaviour or local variability in attractor density of states so that $\mathbb{D} \geq D$. $\mathbb{D}$ can be estimated directly from the time series whereas $D$ can only be measured if we have access to a well-defined attractor (Packard et al. 1980; Ord 1994).

The embedding dimension can be estimated by plotting the number of false neighbours against the embedding dimension (this is done for the Lorentz attractor in Fig. 7c and for GNSS data in Fig. 13). This plot ideally has a minimum at the embedding dimension (Small 2005). In addition, if one defines the correlation dimension, $C_{2}$, for a time series with $\mathrm{N}$ data points:

$$
C_{2}(\mathbb{D}, \varepsilon)=\frac{1}{2 \mathrm{~N}(\mathrm{~N}-\mathrm{T})} \sum_{i} \sum_{j<i-\mathrm{T}} \Theta\left(\varepsilon-\left\|x_{i}-x_{j}\right\|\right)
$$

where $\Theta(*)$ is the Heaviside function, and $\mathrm{T}$ is a parameter large enough that it ensures the distances between points are distributed so that no biases exist towards small numbers, then $C_{2}(\mathbb{D}, \varepsilon)$ represents the fraction, in embedding space, of pairs of points separated by Euclidian distances smaller than $\varepsilon$. A plot of $C_{2}$ against embedding dimension (see Fig. 7b) ideally has an initial slope of $45^{\circ}$ and reaches a plateau at the embedding dimension. Details of methods of estimating the embedding dimension are spelt out by Small (2005) together with the pitfalls involved.

\section{Recurrence plots and recurrence quantification Recurrence plots}

Although nonlinear signal processing is at least 30 years old (Abarbanel 1996; Beck and Schlögl 1995; Sprott 2003; Kantz and Schreiber 2004; Small 2005; Judd and Stemler 2010), most approaches are fairly opaque to potential users. Hence, particularly in the geosciences, the inertia involved in using such developments is very large. However, a step in overcoming the inertia was made by Eckmann et al. (1987) who introduced the concept of recurrence plots which are generalised autocorrelation functions based on Takens' theorem and derived from the conclusion reached by Poincaré (1890) for nonlinear systems that ....., neglecting some exceptional trajectories, the occurrence of which is infinitely improbable, it can be shown, that the system recurs infinitely many times as close as one wishes to its initial state. Since Eckmann's classical paper, the subject has expanded dramatically with important contributions from Casdagli (1997), Webber and Zbilut (2005) and Marwan et al. (2007a). The literature is now very large especially in climate studies, biology and medicine; applications to seismic studies are Chelidze and Matcharashvili (2015) and Garcia et al. (2013) but other applications in the geosciences are rare. Generalised recurrence plots for $n$-dimensional spatial data sets are discussed in Marwan et al. (2007b). If the dimensions of the system are $n$ then the generalised recurrence plot is in $2 n$-space. Thus, a recurrence plot for three-dimensional data exists in 6-space. We only consider one-dimensional data sets in this paper.

A recurrence plot is a symmetrical matrix, $R_{i j}$, expressed as a two-dimensional visualisation and defined by

$$
\mathrm{R}_{\mathrm{ij}}=\Theta\left(\varepsilon-\left\|x_{i}-x_{j}\right\|\right) \text { for } i, j=1, \mathrm{~N}
$$

where $\varepsilon$ is an arbitrary threshold distance (commonly called the radius) that measures the tolerance within which recurrence is identified and $\left\|^{*}\right\|$ denotes a norm, commonly taken as the Euclidean norm. (4) says that we measure the distance between a given point, $x_{i}$, on the signal and every other point, $j=1$ to $\mathrm{N}$ on the signal and give that measure the value 1 if the distance is within the tolerance, $\varepsilon$, or zero if not. This is repeated for all values of $i=1$ to $\mathrm{N}$ to form the recurrence matrix. The caveat is that the distances are measured in the embedding space. A recurrence plot is then a plot of $\mathrm{R}_{i j}$ for a given radius, $\varepsilon$. If $\left\|x_{i}-x_{j}\right\| \approx \varepsilon$ then $\mathrm{R}_{i j}=1$ and a dot is added to the plot; otherwise, $\mathrm{R}_{i j}=0$ and the plot are left blank. The plot may be contoured by setting $\left\|x_{i}-x_{j}\right\| \approx$ $0.8 \varepsilon, 0.6 \varepsilon, \ldots 0.2 \varepsilon$ and so on. In some software (VRA), the contour interval is prescribed within the software; for others, a number, $c$, can be set which defines the number of contours with equal spacing, $(\varepsilon / c)$. We repeat, a recurrence plot is a generalised autocorrelation function.

If the signal represents white noise then the embedding dimension is infinite and recurrences occur randomly with any patterning in the recurrences occurring by chance. Multiple recurrence plots of the data will not be reproducible; the lack of any diagonal lines indicates that the signal is completely stochastic. An example is shown in Fig. 1a. If the signal is a sine-wave then recurrences occur with a period equal to that of the signal; the recurrence plot comprises continuous diagonal lines as shown in Fig. 1b with the vertical (or horizontal) distance between diagonals equal to the period. The continuity of the diagonal lines indicates that the signal is completely deterministic. The addition of white noise to a sine-wave signal results in blurring of the recurrence plot but the basic pattern and determinism are well 

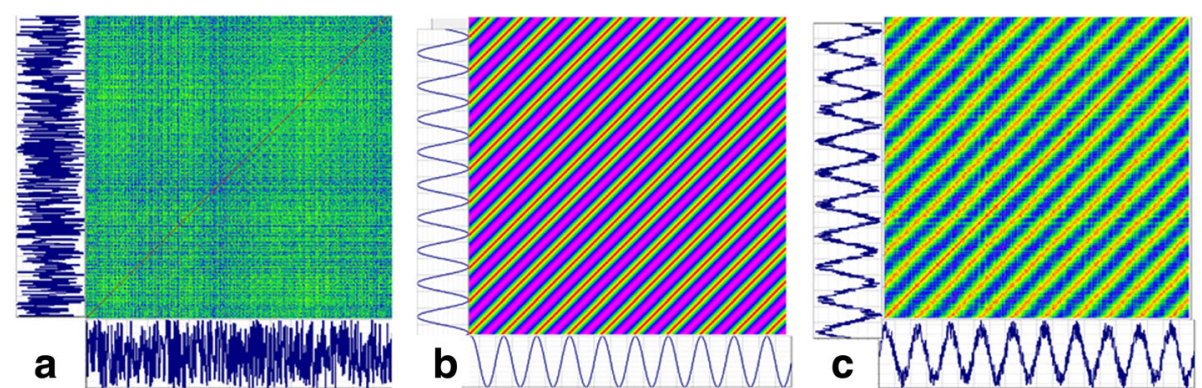

Fig. 1 Examples of recurrence plots. a White noise. Any patterning occurs by chance. b A sine-wave with no noise. The vertical (or horizontal) distance between red lines is the period of the signal. c A sine-wave with noise. The signal is blurred and there is a faint underlying patterning of horizontal and vertical lines but the overall pattern of diagonal lines is preserved

preserved (Fig. 1c). Recurrence plots are applicable to stationary and nonstationary data sets and are reasonably insensitive to noise. Pitfalls associated with recurrence analysis are discussed by Marwan (2011).

\section{Recurrence quantification}

Although recurrence plots make "pretty pictures" (especially if $\varepsilon$ is large and $c$ is small) and are useful for comparing and visually classifying different data sets, the real power lies in the quantitative measures, including the invariant measures, that can be derived from them. In principle, because of Takens' theorem, all of the dynamics of the system are encoded in the recurrence plot and the quantitative measures are designed to expose the dynamics. Recurrence quantification analysis (RQA) is based on deriving quantitative information from the distribution of lines on the recurrence plot. For a detailed discussion of these measures, see Webber and Zbilut (2005) and Marwan et al. (2007a); in Table 1, we present a summary of many of these measures. Figure 2 shows a summary of many of the steps involved in, and outputs from, an RQA and we will elaborate upon this diagram later in the paper with respect to GNSS data sets.

The recurrence plots shown in Fig. 1 are relatively simple and represent completely stochastic systems (Fig. 1a) or completely deterministic systems (Fig. 1b). Most natural systems lie somewhere between these two extremes. An example is given in Fig. 3 which is the recurrence plot for one of the time series from the Lorentz system (Sprott 2003) examined in greater detail later in the paper. The recurrence plot, which here has been constructed to emphasise the main features in typical recurrence plots, consists of many vertical and horizontal lines as well as diagonal lines. Below, we consider the significance of these lines; they are the basis for RQA.

Note that two invariant measures may be derived from the recurrence plot. The entropy is given by ENT: for periodic signals, ENT $=0$ bits/bin, and for the Hénon attractor (Sprott 2003, p. 421), ENT = 2.557 bits/bin (Webber and Zbilut 2005). The first positive Lyapunov exponent is proportional to (1/ DMAX). The smaller DMAX, the more chaotic is the

Table 1 Summary of quantities used in recurrence quantification analysis. Modified after Webber and Zbilut (2005): https:// www.nsf.gov/pubs/2005/nsf05057/nmbs/nmbs.pdf

\begin{tabular}{|c|c|c|}
\hline \%recurrence, \%REC & Percentage of recurrent points falling within the specified radius, $\varepsilon$. & $\% \mathrm{REC}=100 \frac{\text { number of points in triangle }}{\varepsilon(\varepsilon-1) / 2}$ \\
\hline $\begin{array}{l}\text { \%determinism, } \\
\text { \%DET }\end{array}$ & $\begin{array}{l}\text { Percentage of recurrent points forming diagonal line structures. } \\
\text { This is a measure of determinism in the signal. }\end{array}$ & $\% \mathrm{DET}=100 \frac{\text { number of points in diagonal lines }}{\text { number of recurrent points }}$ \\
\hline Linemax, DMAX & $\begin{array}{l}\text { The length of the longest diagonal line in the plot } \\
\text { (except main diagonal). }\end{array}$ & $\begin{array}{l}\text { DMAX = length of longest diagonal line in the recurrence } \\
\text { plot }\end{array}$ \\
\hline Entropy, ENT & $\begin{array}{l}\text { The Shannon information entropy of all diagonal line lengths } \\
\text { over integer bins in a histogram. This is a measure of signal } \\
\text { complexity with units bits/bin. }\end{array}$ & $\mathrm{ENT}=-\sum\left(P_{\text {bin }}\right) \log _{2}\left(P_{\text {bin }}\right)$ \\
\hline Trend. TND & A measure of system stationarity. & $\mathrm{TND}=1000\left(\begin{array}{c}\text { slope of \%local recurrence } \\
\text { vs.displacement }\end{array}\right)$ \\
\hline \%laminarity, \%LAM & $\begin{array}{l}\text { The percentage of recurrent points forming vertical line } \\
\text { structures. }\end{array}$ & $\% \mathrm{LAM}=100 \frac{\text { number of points in vertical lines }}{\text { number of recurrent points }}$ \\
\hline VMAX & The length of the longest vertical line in the plot. & $\mathrm{VMAX}=$ length of longest vertical line in the recurrence plot \\
\hline Trapping time, $\Pi$ & The average length of vertical line structures. & $\Pi=$ Average length of vertical lines $\geq$ parameter line \\
\hline
\end{tabular}




\section{RECURRENCE NONLINEAR ANALYSIS}

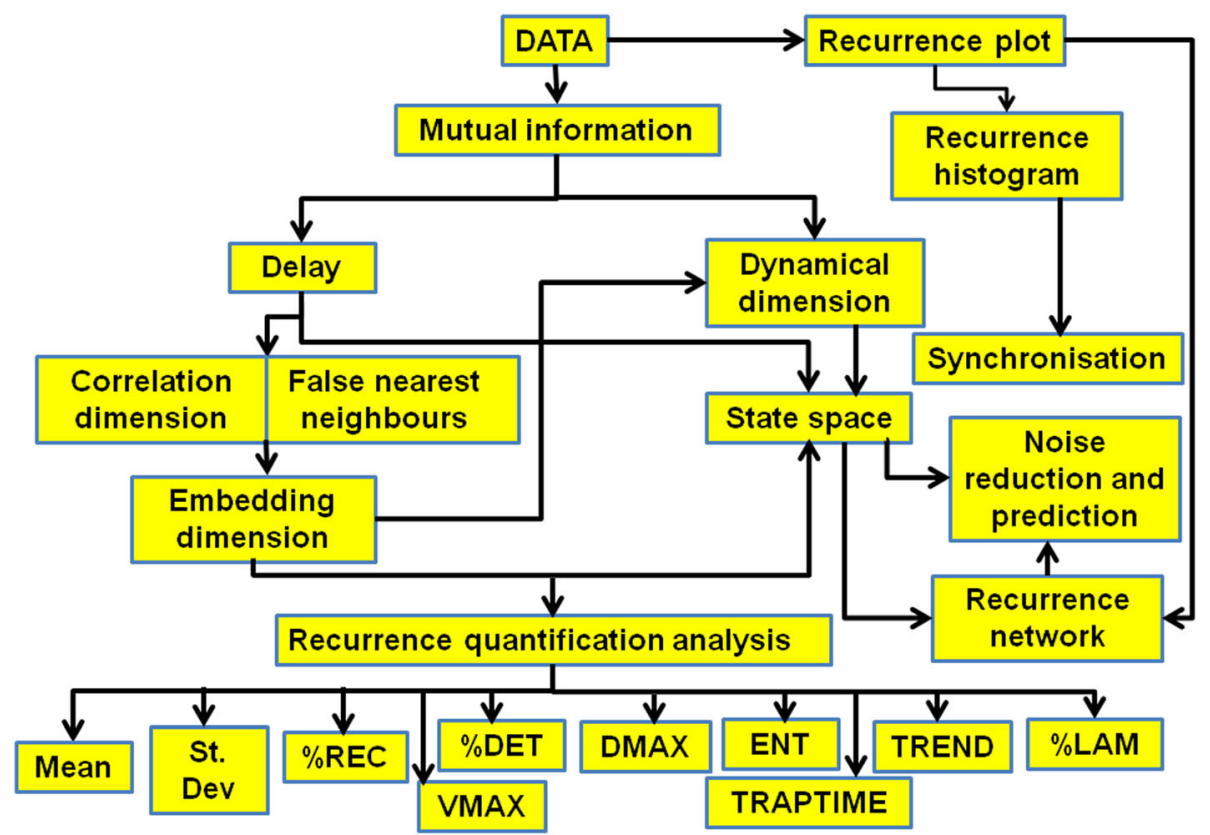

Fig. 2 Recurrence quantification analysis (RQA). This diagram shows the steps typically taken in a recurrence nonlinear analysis of a time series together with many of the outputs from the RQA. Modified from Aks (2011)

signal. For the Hénon attractor, DMAX $=12$ points (Webber and Zbilut 2005).

In addition to the diagonal lines in Fig. 3 which are related to recurrence and determinism, vertical (and horizontal) lines appear on many recurrence plots. These mark transitions in the behaviour of the system. Such transitions may be periodic $\rightarrow$ periodic (with a change in frequency), periodic $\rightarrow$ chaotic or chaotic $\rightarrow$ chaotic. A vertical line represents an interval where the state does not change or changes relatively slowly but the state of the system changes across the line. A summary of the significance of various patterns on recurrence plots is given in Table 2.

\section{First Lyapunov exponent $=(1 /$ Length of longest diagonal line $)$}

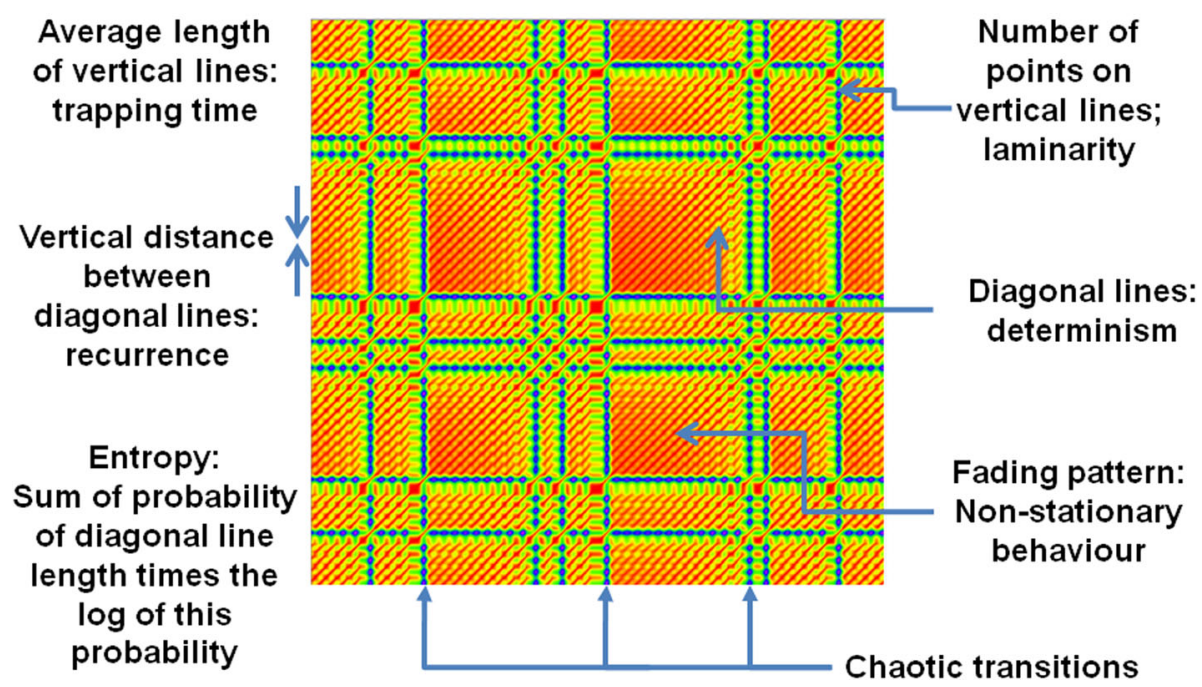

Fig. 3 A recurrence plot for the Lorentz attractor showing the significance of some of the RQA measures and of other features of the plot 
Table 2 Significance of patterns in recurrence plots (after Marwan et al. 2007a)

\begin{tabular}{|c|c|}
\hline Pattern & Significance \\
\hline Homogeneous & The process is stationary \\
\hline $\begin{array}{l}\text { Fading pattern to upper right or lower } \\
\text { left }\end{array}$ & Non-stationary data; the process contains a trend or drift \\
\hline Disruptions (horizontal or vertical) & Non-stationary data; some states are far from the normal; transitions may have occurred \\
\hline Periodic or quasi-periodic patterns & $\begin{array}{l}\text { The process is cyclic. The vertical (or horizontal) distance between periodic lines corresponds to the period. } \\
\text { Variations in the distance mean quasi-periodicity in the process. }\end{array}$ \\
\hline Single isolated points & Strong fluctuations in the process. The process may be uncorrelated or anti-correlated. \\
\hline Diagonal lines (parallel to the LOI) & $\begin{array}{l}\text { The evolution of the system is similar over the length of the line. If lines appear next to single isolated } \\
\text { points the process may be chaotic. }\end{array}$ \\
\hline Diagonal lines (orthogonal to the LOI) & The evolution of states at different times is similar but with reverse timing. \\
\hline Vertical and horizontal lines or clusters & States do not change with time or change slowly \\
\hline $\begin{array}{l}\text { Lines not parallel to the LOI-sometimes } \\
\text { curved. }\end{array}$ & $\begin{array}{l}\text { The evolution of states is similar at different times but the rate of evolution changes with time. The } \\
\text { dynamics of the system is changing with time. }\end{array}$ \\
\hline
\end{tabular}

Recurrence plots and their quantification in this paper have been prepared using the software VRA: http:// visual-recurrence-analysis.software.informer.com/4.9/ and RQA software: http://cwebber.sites.luc.edu/. Other software is available at http://tocsy.pik-potsdam.de/, http:// tocsy.pik-potsdam.de/CRPtoolbox/, http://tocsy.pik-potsdam.de/pyunicorn.php and https://www.pks.mpg.de/ $\sim$ tisean/.

\section{Prediction and noise reduction}

Most signals, especially those from natural systems, contain some form of noise which consists of the addition of a stochastic (originates from uncorrelated processes) signal. It is generally considered as an adulteration to the signal and needs to be removed or reduced as far as possible. This notion arises from a linear view of the world where the solutions to linear differential equations are smoothly varying functions and any irregularity must be the result of externally imposed random input. However, irregular behaviour including non-periodicity and intermittency can arise from nonlinear systems with no externally imposed noise. The problem that arises in nonlinear systems is to understand if some of the noise results from deterministic processes of interest and hence should be retained.

Noise is generally classified as white noise with no long-range correlations and coloured noise with some internal structure and long-range correlations (Moss and McClintock 1989). In addition, two different sources of noise can be identified (Grassberger et al. 1993; Kantz 1994; Judd and Stemler 2009). If the behaviour of the system can be expressed at discrete intervals (as in a GNSS lithospheric deformation system) then the sequence of states, $z_{t}$, at times, $t$, can be written

$$
z_{t+1}=f\left(z_{t}\right)+v_{t}
$$

where the function $f$ defines the dynamics of the system (generally written as set of coupled partial differential equations) and expresses the way in which the system evolves due to deterministic processes and $v_{t}$ are a series of independent random variables arising from some process operating in the system. This is referred to as dynamical noise.

Dynamical noise might be generated in a system where different processes dominate at different time and/or length scales so that some frequencies and/or parts of the system evolve in different ways and rates to others. This results in probability distributions for some time/ length scales diffusing (broadening) and drifting (shifting the mean) with different diffusivities as described by Fokker-Planck equations (Moss and McClintock 1989). Such processes add a stochastic but dynamic noise to the system behaviour but such noise is a fundamental part of the processes operating in the system and should be preserved in any noise reduction algorithm. This kind of noise is generally, but not always, coloured noise (Moss and McClintock 1989).

If we make observations, $s_{t}$, at discrete intervals (as in GNSS time series) then

$$
s_{t+1}=g\left(s_{t}\right)+\varepsilon_{t}
$$

where $g$ expresses the state of the system at time $t$ and $\varepsilon_{t}$ are independent random variables arising from processes external to the system and comprise observational noise. Such noise may be white or coloured. Any noise reduction process should attempt to reduce the contribution from observational noise whilst preserving as much as possible of the dynamical noise. Later in the paper, we give examples of noise reduction for GNSS data and show that some methods preserve the RQA measures of the signal whereas others degrade some deterministic aspects of the signal. 
To a large extent, the process of nonlinear noise reduction is the inverse of the nonlinear prediction (or forecasting) problem. For nonlinear noise reduction, one can determine the dynamics of the system given the whole signal up to its current state and then search for parts of the signal in the past that are not part of the dynamics. These parts are removed as noise. This means we take the whole signal and work backwards. For prediction, we take one part of the signal, determine the dynamics and then see if we can find a part of the dynamics that fits the way in which the signal is evolving into the future and use that to make a forward prediction or forecast. Nonlinear prediction is particularly useful if one needs to "fill in" short gaps in data sets in a manner that honours the deterministic dynamics of the system.

An approach to prediction in chaotic systems is spelt out here based on Casdagli and Eubank (1992), Weigend and Gershenfeld (1994), Fan and Gijbels (1996) and Abarbanel (1996). To predict a point $x_{n+1}$, we determine the last known state of the system as represented by the vector $\mathbf{X}=\left[x_{n}, x_{n-\tau}, x_{n-2 \tau}, \ldots \ldots ., x_{n-(\mathbb{D}-1) \tau}\right]$, where $\mathbb{D}$ is the embedding dimension and $\tau$ is the delay. We then search the series to find $k$ similar states that have occurred in the past, where "similarity" is determined by evaluating the distance between the vector $\mathbf{X}$ and its neighbour vector $\mathbf{X}^{\prime}$ in the $D$-dimensional state space. The concept is that if the observable signal was generated by some deterministic map, $\mathrm{M}:\left(\ldots . .\left(\left(x_{n}, x_{n-\tau}\right), x_{n-2 \tau}\right), \ldots, x_{n-(\mathbb{D}-1) \tau}\right.$ )$=x_{n+1}$; that map can be reconstructed from the data by looking at the signal behaviour in the neighbourhood of $\mathbf{X}$. We find the approximation of $\mathrm{M}$ by fitting a low-order polynomial (Fan and Gijbels 1996) which maps $k$ nearest neighbours (similar states) of $\mathbf{X}$ onto their next immediate values. Now, we can use this map to predict $x_{n+1}$. In other words, we make an assumption that $\mathrm{M}$ is fairly smooth around $\mathbf{X}$, and so if a state $\mathbf{X}^{\prime}=$ [ $\left.x_{n}^{\prime}, x_{n-\tau}^{\prime}, x_{n-2 \tau}^{\prime}, \ldots, x_{n-(\mathbb{D}-1) \tau}^{\prime}\right]$ in the neighbourhood of $\mathbf{X}$ resulted in the observation, $x_{n+1}^{\prime}$, in the past, then the point $x_{n+1}$ which we want to predict must be somewhere near $x_{n+1}^{\prime}$. In any chaotic system, we expect the error in prediction to increase exponentially (as measured by the Lyapunov exponent) as we move away from known data.

The above approach is based on intensive work on prediction in chaotic systems largely carried out in the 1990s and relies on finding local states in the past that resemble current states of the system. A relatively recent approach to nonlinear filtering is the shadowing filter (Stemler and Judd 2009). A shadowing filter (Davies 1993; Bröcker et al. 2002; Judd 2003; Judd 2008a, 2008b) searches in state space for a trajectory (defined by a sequence of $z_{t}$ for the system), rather than local states, that remains close to (that is, the trajectory shadows) a sequence of observations, $s_{t}$, on the system. The algorithm is discussed by Judd and Stemler (2009). We do not use a shadowing filter in this paper, but its use in future work promises to give better results than reported here.

\section{Synchronisation}

Of particular interest in systems where many coupled episodic sub-systems are operating, such as in GNSS and seismic systems, is to see if the sub-systems influence each other so that some form of spatial or temporal synchronisation occurs. Such synchronisation can be of five forms (Romano Blasco 2004; Marwan et al. 2007a):

- Phase synchronisation: the two signals are phase locked but amplitudes are not identical.

- Frequency synchronisation: the two signals are frequency locked.

- Lag synchronisation: there is a time or space lag between similar or identical states.

- Generalised synchronisation: the synchronisation comprises nonlinear locking between similar or identical states.

- Chaotic transition synchronisation: similar behaviour in the signal is locked into chaotic transitions in the respective recurrence plots that occur at similar times in two or more time series.

In many systems, synchronisation switches from one of these five types to another as the system evolves and the coupling between parts of the system changes strength (Romano Blasco 2004). We will see that cross recurrence plots and particularly joint recurrence plots are powerful ways of investigating such synchronisation (Marwan et al. 2007a). Just as a recurrence plot identifies recurrences at different parts of the same signal, cross recurrence plots identify recurrences at identical times on two different signals. In other words, a cross recurrence plot identifies those times when a state in one system recurs in the other. Joint recurrence plots identify recurrences in the recurrence histograms of two signals; they are somewhat similar to identifying simultaneously occurring maxima in power spectra from two different signals in linear systems. Clearly, the plots only reflect something of the dynamics if both signals originate from similar processes and belong to state spaces with similar or identical attractors.

By analogue with (4) a cross recurrence matrix for two time series $x_{i}$ and $y_{j}$ is defined as

$$
\mathrm{CR}_{\mathrm{ij}}=\Theta\left(\varepsilon-\left\|x_{i}-y_{j}\right\|\right) \text { for } i=1, \mathrm{~N} \text { and } j=1, \mathrm{M}
$$

A cross recurrence plot is a generalisation of a linear cross correlation function. Additionally, we define a joint recurrence matrix as 


$$
\mathrm{JR}_{\mathrm{ij}}=\Theta\left(\varepsilon^{x}-\left\|x_{i}-x_{j}\right\|\right) \Theta\left(\varepsilon^{y}-\left\|y_{i}-y_{j}\right\|\right) \quad \text { for } i, j=1, \mathrm{~N}
$$

where $\varepsilon^{x}$ and $\varepsilon^{y}$ are tolerances for the individual time series. For joint recurrence, $\mathrm{JR}_{i j}=1$ if $\left\|x_{i}-x_{j}\right\|<\varepsilon^{x}$ and $\left\|y_{i}-y_{j}\right\|<\varepsilon^{y}$ otherwise $\mathrm{JR}_{i j}=0$. Joint recurrence measures the probability that both the $x_{i}$ and $y_{j}$ systems revisit simultaneously the neighbourhood of a point in their respective phase spaces previously revisited The equivalent of an RQA analysis can be conducted for each of these matrices, CRQA and JRQA respectively but in some instances the measures may have limited use (Romano Blasco 2004). For instance in a cross recurrence plot for two signals with different frequencies (see Fig. 4b below), there may be no diagonal lines so that measures such as \%DET and DMAX have little meaning. Romano Blasco (2004) shows that the entropy in particular is a useful measure for studying synchronisation in joint recurrence plots. In general, cross recurrence is not a useful way of analysing synchronicity between two systems (Romano Blasco 2004) but we use aspects of cross recurrence plots below as a useful way of portraying synchronisation between signals from two GNSS stations.

In Fig. 4, we show several different cross and joint recurrence plots so that the reader might obtain some insight into how to read such plots. In Fig. 4a recurrences between the signals $y=\sin (x)$ and $y=\cos (x)$ are plotted. The recurrences plot on straight diagonal lines and the vertical distance between these lines is the (identical) period of both signals. The straight diagonal lines are referred to (Marwan et al. 2007a) as lines of identity (LOI). In a more general recurrence plot for a dynamical system, the LOIs represent segments of the trajectories of both systems that run parallel for some time. The frequency and lengths of these lines are measures of the similarity and nonlinear interactions between the two systems.

Figure $4 \mathrm{~b}$ is a cross recurrence plot between the two signals $y=\sin (x)$ and $y=\sin (2 x)$. The LOIs are now inclined at $\beta=\tan ^{-1}(1 / 2)=26.6^{\circ}$ to the horizontal axis. The slope, $\beta$, is given by Marwan et al. (2007a)

$$
\beta=\tan ^{-1}\left\{\frac{\partial}{\partial t}\left(\frac{T_{1}}{T_{2}}\right)\right\}
$$

where $T_{1}$ and $T_{2}$ are the time scale characteristic of the two systems.

In Fig. 4c, recurrences between $y=\sin (x)$ and $y=$ $\sin (x)+\sin \left(5 x^{2}\right)$ are plotted. The straight LOIs of Fig. $4 \mathrm{a}$, $\mathrm{b}$ are now curved and are referred to as lines of synchronisation (LOSs). Thus, the details of the cross recurrence plot can give information on whether the signals that are compared are linear or nonlinear and also give an indication of both the absolute and the relative time scales associated with the two systems. Figure $4 \mathrm{~d}$ is a cross recurrence plot between the two quasi-periodic signals: $y=\sin (x)+\sin (\sqrt{2} x)$ and $y=\sin (x)+\sin (\pi x)$. Figure $4 \mathrm{e}$ is a cross recurrence plot between two logistic signals given by $x_{n+1}=\alpha x_{n}\left(1+x_{n}\right)$ with $\alpha=3.7$ and 3.8 and Fig. $4 \mathrm{f}$ is a joint recurrence plot between the signals: $y=\sin (x)$ and $y=\sin (20 x)$.

Plotting the changes in slopes of LOSs is a powerful way of tracking the evolution of two synchronised systems and of observing the ways in which time scales that characterise each system change with time.

Examples of joint recurrence plots are given in Fig. 4g, $\mathrm{h}$, i for the same signals in the cross recurrence plots of Fig. 4a, b, c. In contrast to the cross recurrence plots (a to c) which express the ways in which two signals occupy similar states synchronously, a joint recurrence plot expresses (in the form of blue lines or dots in $g$ to i) the ways in which recurrences on two different signals occur synchronously.

\section{An example: the Lorentz attractor-quantification and prediction}

As an example of the principles involved in nonlinear analysis and prediction, we present a discussion centred on the relatively simple, low-dimensional Lorentz attractor (Sprott 2003, pp. 90-92) which forms the basis for modern weather forecasts (Yoden 2007). This system is described by the set of differential equations:

$$
\begin{aligned}
& \frac{d x_{1}}{d t}=-\frac{3}{2} P x_{1}+\frac{2}{3} a P x_{2} \\
& \frac{d x_{2}}{d t}=a x_{1} x_{3}-\frac{3}{2} x_{2}+a R x_{1} \\
& \frac{d x_{3}}{d t}=-\frac{1}{2} a x_{1} x_{2}-4 x_{3}
\end{aligned}
$$

where $x_{1}, x_{2}$ and $x_{3}$ are variables of interest, $t$ is time and $a, P$ and $R$ are parameters of the system. The signal that results for $x_{1}$ from these equations with $a=2.25, P$ $=20 / 3$ and $R=-4 / 9$ is given in Fig. $5 \mathrm{a}$ with the multifractal spectrum in Fig. 5b. The well-defined multifractal spectrum arises because the Lorentz system is chaotic and, in principle, its attractor consists of an indefinite number of singularities with variable densities of occurrence, $\alpha$, on the attractor (Beck and Schlögl (1995). The multifractal spectrum expresses the density distribution, $f(\alpha)$, of these singularities as a function of their strength, $\alpha$ (Arneodo et al. 1995).

The attractor formed by plotting one variable against another is shown in Fig. 6 in two dimensions. The recurrence plot is shown in Fig. 7a for 3500 points in the time series. The dimensions of the space in which the attractor lives (the embedding dimension) is estimated from the two plots: the correlation dimension against 

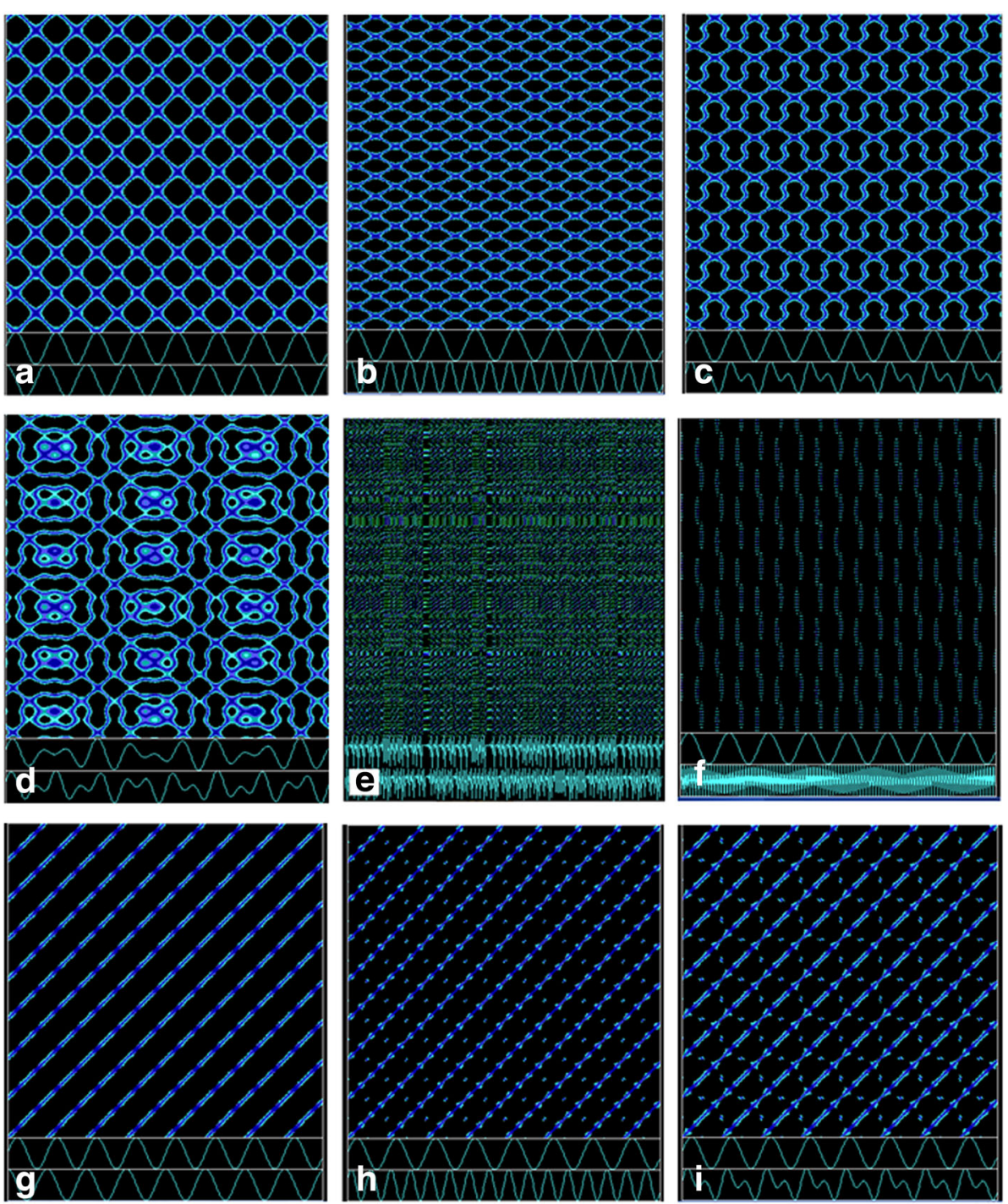

Fig. 4 Cross and joint recurrence plots. a Cross recurrence plot between $\sin (x)$ (upper trace) and $\cos (x)$ (lower trace). High recurrence is represented by the dark blue (straight) lines of synchronisation at $45^{\circ}$ to the horizontal axis. $\mathbf{b}$ Cross recurrence plot between $\sin (x)$ (upper trace) and $\sin (2 x)$ (lower trace). High recurrence is represented by the dark blue (straight) lines of synchronisation $\operatorname{tat}^{-1} \tan ^{-1}(1 / 2)=26.6^{\circ}$ to the horizontal axis. The ratio $1 / 2$ is the ratio of the frequencies of the two signals. c Cross recurrence plot between $\sin (x)$ (upper trace) and $\sin (x)+\sin \left(5 x^{2}\right)$ (lower trace). High recurrence is represented by the (curved) dark blue lines of synchronisation. The local slope of the line of synchronisation is the arctangent of the ratios of the local frequencies of the signals. $\mathbf{d}$ Cross recurrence between two quasi-periodic signals: $y=\sin (x)+\sin (\sqrt{2} x)$ (upper frame), $y=\sin (x)+\sin (\pi x)$ (lower frame). e Cross recurrence plot between signals from two logistic equations, $x_{n+1}=a x_{n}\left(1-x_{n}\right)$, with $a=3.7$ (upper frame) and $a=3.8$ (lower frame). The cross recurrence plot is dominated by chaotic transitions (vertical and horizontal lines) but regions of periodic behaviour occur characterised by equally spaced diagonal lines. $f$ Cross recurrence plot for $y=\sin (x)$ (upper frame) and $y=\sin (20 x)$ (lower frame). The large frequency difference between the two signals means that the LOSs are almost vertical. $\mathbf{g}$ Joint recurrence plot between $\sin (x)$ (upper trace) and $\cos (x)$ (lower trace). High synchronisation of recurrences is represented by the dark blue regions of synchronisation. $\mathbf{h}$ Joint recurrence plot between $\sin (x)$ (upper trace) and $\sin (2 x)$ (lower trace). Lines of high synchronisation between recurrences are represented by the dark blue lines. $\mathbf{i}$ Joint recurrence plot between sin $(x)$ (upper trace) and $\sin (x)+\sin \left(5 x^{2}\right)$ (lower trace). Again high synchronisation of recurrences is represented by the dark blue lines

embedding dimension (Fig. 7b) and the percentage of false neighbours against embedding dimension (Fig. 7c). The estimated dynamical dimension for the Lorentz attractor is 2.1736 whereas Fig. $7 \mathrm{~b}$, c would suggest an embedding dimension of $\approx 2$. Thus, it is not necessary for the attractor to be known for a system, its embedding dimension and its topology can be estimated from the recurrence plot. This dimension is important for constraining any data model that is constructed.

Although we will not explore recurrence networks in this paper we mention, for completeness, that a network with the topology of the attractor can be calculated from the recurrence plot (Donner et al. 2010; McCullough et al. 2017) as shown in Fig. 8. The adjunct matrix, $\mathrm{A}_{i j}$, for 

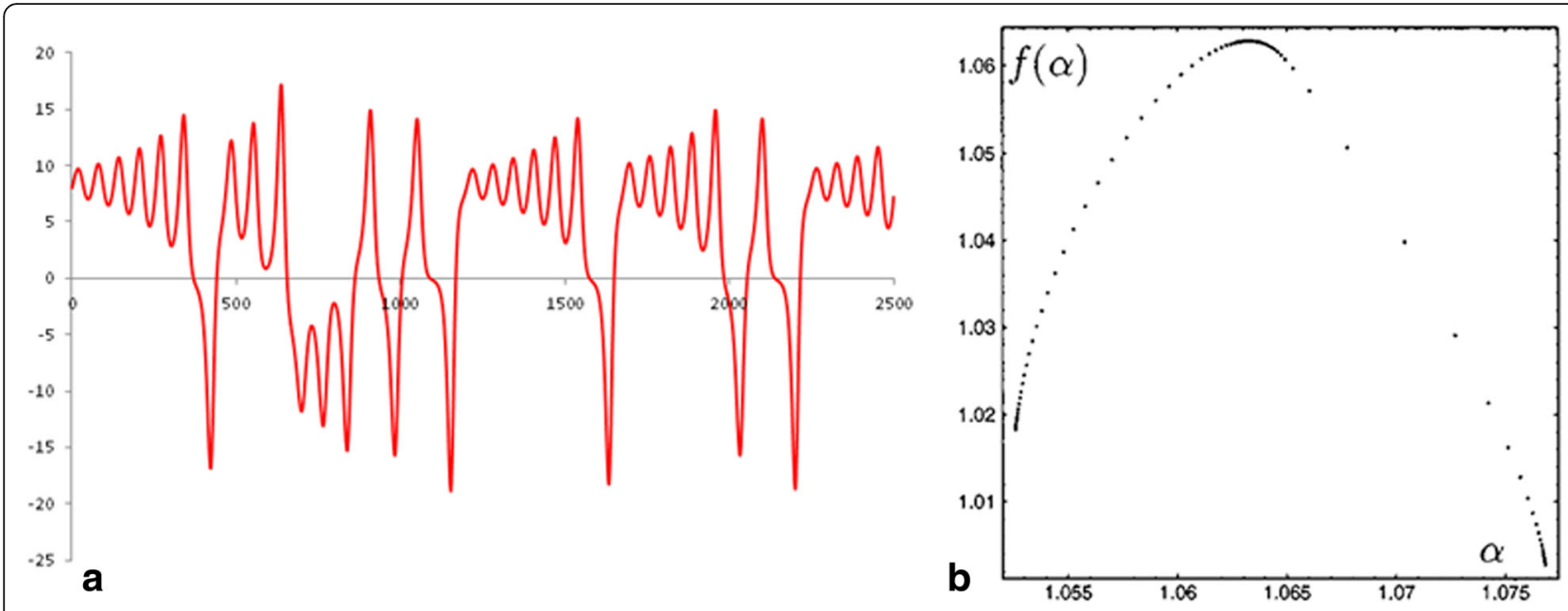

Fig. 5 Features of the Lorentz system. a $x_{1}$ signal from Lorentz signal with parameters given in the text. b Multifractal spectrum from Lorentz system

the network is related to the recurrence matrix in (4) by $\mathrm{A}_{i j}=\mathrm{R}_{i j}-\delta_{i j}$ where $\delta_{i j}$ is the Kronecker delta. Recurrence network software is available at http://tocsy.pik-potsdam.de/pyunicorn.php and needs to be used in conjunction with a graphical network code such as Gephi https://gephi.org/. Recurrence networks have been applied to seismic time series by Lin et al. (2016). We revisit the concept of nonlinear networks in the "Discussion" section as a basis for monitoring the behaviour of GNSS data networks.

The application of nonlinear noise reduction to the Lorentz system is shown in Fig. 9 where one can see that the prediction is still very good 450 steps away from a 3000 -step training set but is rising exponentially by the end of the prediction. In plots such as these, the normalised error is that relative to that which would be expected from a linear prediction of the mean expressed as the normalised mean squared error, NMSE, discussed in Weigend and Gershenfeld (1994) and defined as

$$
\mathrm{NMSE}=\frac{1}{\sigma^{2} N} \sum_{i=1}^{N}\left(x_{i}-\hat{x}_{i}\right)^{2}
$$

where $x_{i}$ is the observed value of the $i^{\text {th }}$ point in a series of length $N, \hat{x}_{i}$ is the predicted value and $\sigma$ is the standard deviation of the observed time series over the length, $N$. NMSE is the ratio of the mean squared errors of the prediction method used to a method that predicts the mean at every step. In Fig. 9, this ratio is a maximum of $\approx 0.0065$.

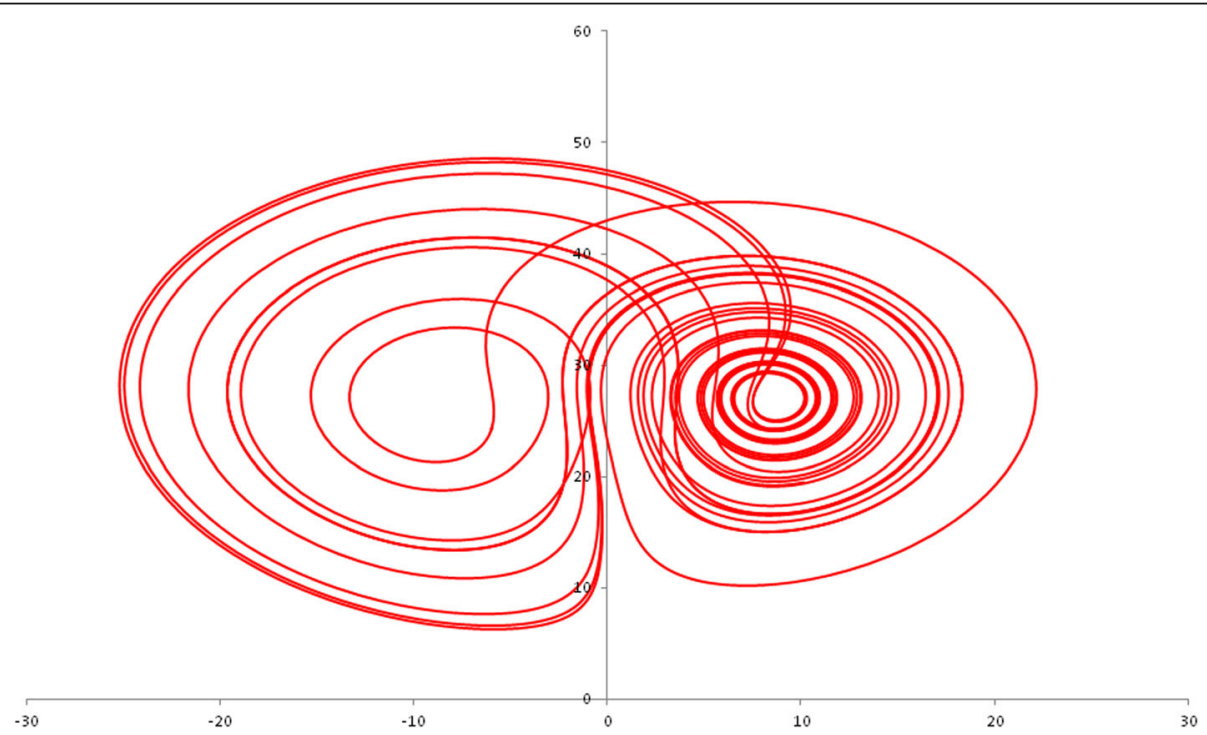

Fig. 6 Attractor for the Lorentz system, $x_{2}$ plotted against $x_{3}$ 


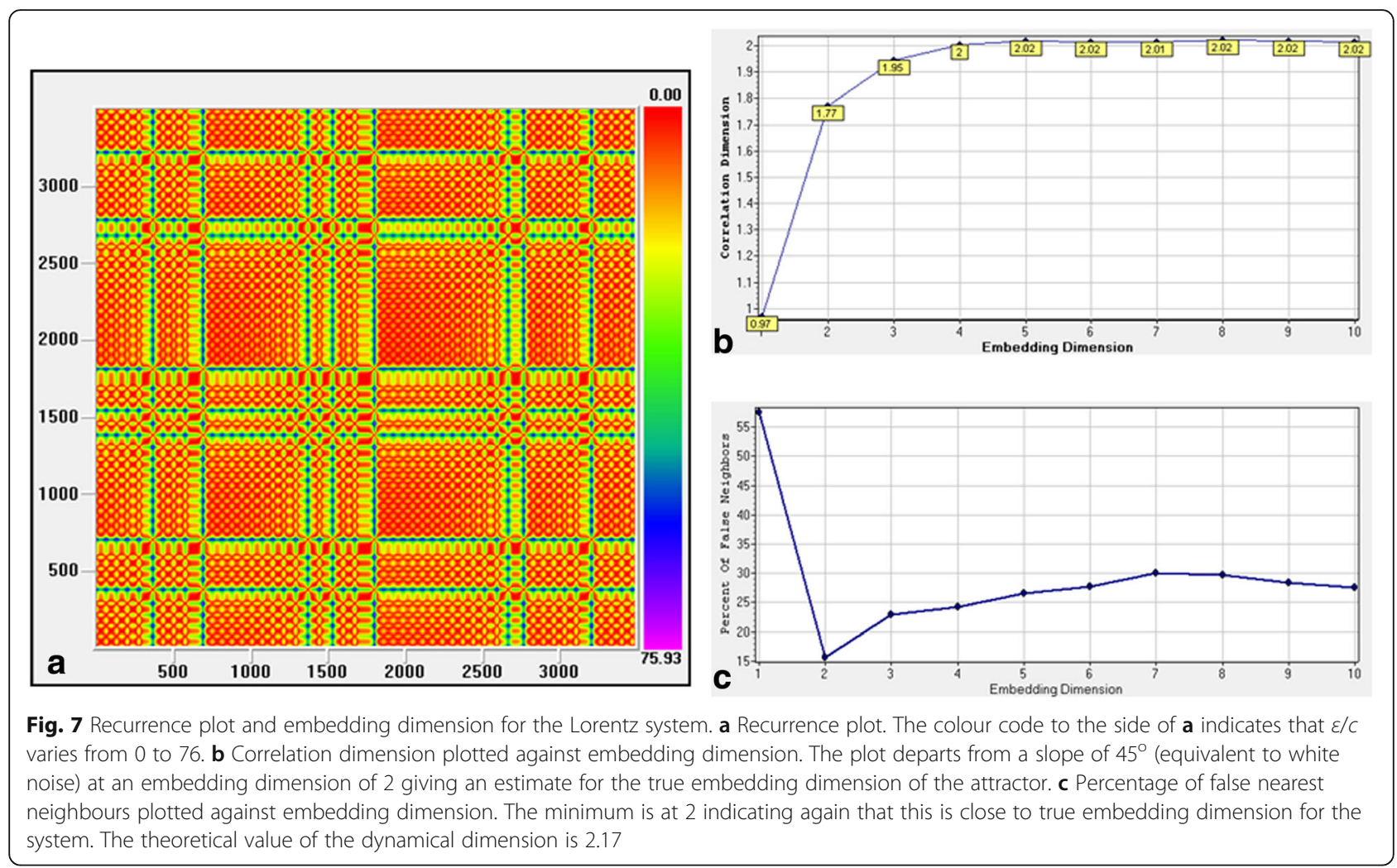

\section{Significance of a linear trend in the data}

An issue that arises in the nonlinear analysis of GNSS data is the significance of any linear trend that commonly exists in the data and of the effect of removing this trend. Consider a pair of time series, $x_{i}$ and $y_{j}$, with $i=1, \mathrm{~N}$ and $j=1, \mathrm{M}$ and a pair of derived time series, $\hat{x}_{i}, \hat{y}_{j}$, where $\hat{x}_{i}=x_{i}+a t$ and $\hat{y}_{i}=y_{i}+b t$ where $a$ and $b$ are constants and $t$ is time. Then, $x_{i}, y_{j}$ are time series derived from $\hat{x}_{i}, \hat{y}_{j}$ by removal of a linear trend. For the cross recurrence plots of these pairs of time series to be identical,

$$
\Theta\left(\varepsilon-\left\|x_{i}-y_{j}\right\|\right)=\Theta\left(\varepsilon-\left\|\hat{x}_{i}-\hat{y}_{j}\right\|\right) \text { for } i=1, \mathrm{~N} \text { and } j=1, \mathrm{M}
$$

This condition can be satisfied if $\left(\left\|x_{i}-y_{j}\right\|\right)=\left(\left\|\hat{x}_{i}-\hat{y}_{j}\right\|\right)$ for all $i=1, \mathrm{~N}$ and $j=1, \mathrm{M}$ but in general this will be a difficult condition to satisfy. Similarly joint recurrence

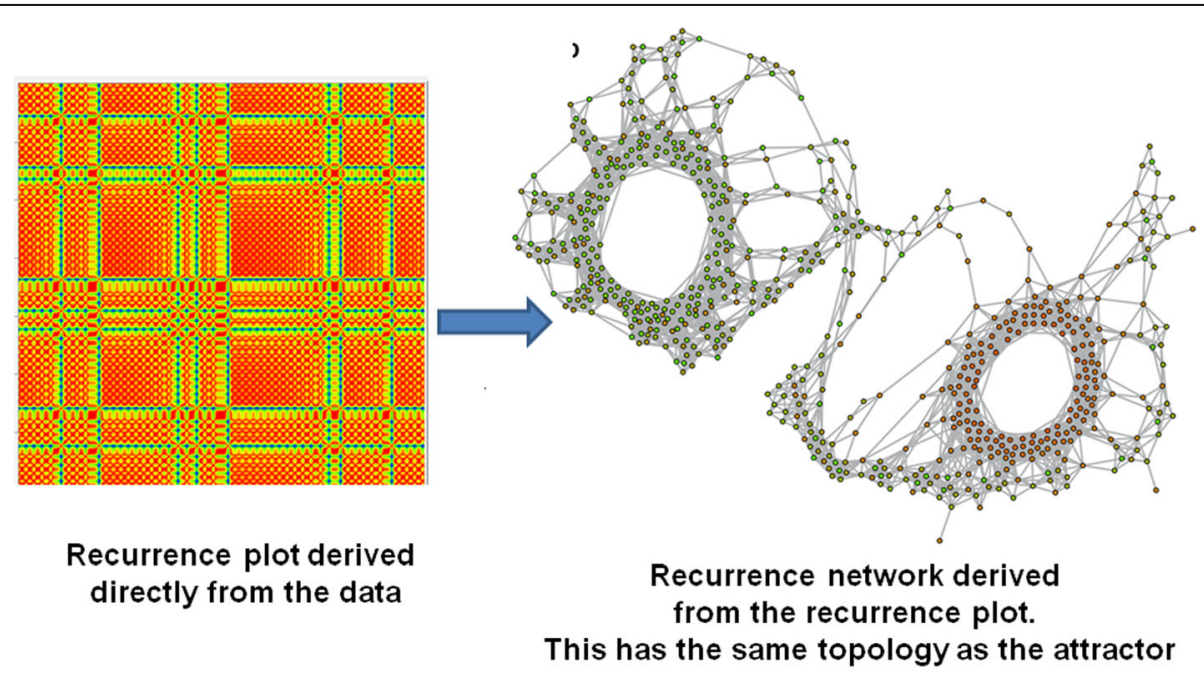

Fig. 8 Recurrence network for the Lorentz system. The recurrence network has the same topology as the attractor (Fig. 6) 


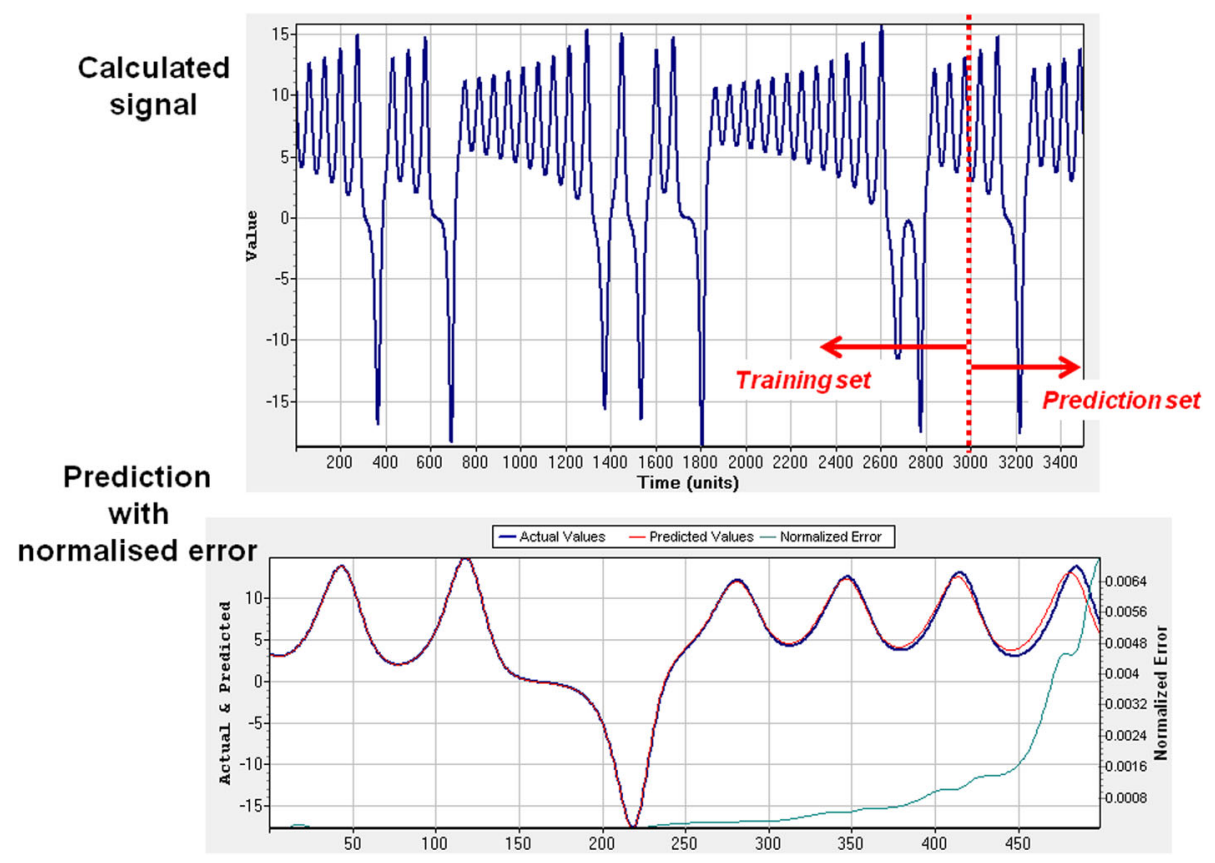

Fig. 9 Prediction in the Lorentz system. The top panel is the signal from the Lorentz system calculated from the differential equations that describe the system. We use the first 3000 steps as a training set for a nonparametric prediction over the next 500 steps in the range 3001 to 3500 steps shown in the lower panel. One can see that the prediction (in red) hugs the real signal (in black) fairly well over the first 250 steps of the prediction (normalised error $<0.0008$ ). The error is normalised relative to the prediction obtained from a linear prediction or random walk model. From then on the error begins to rise exponentially (as is to be expected from a chaotic series) and is 0.0064 (or very close to $100 \%$ of the variance in the data) at 500 steps. If one wanted to improve the accuracy of the prediction past this range then the collection of data within those last 250 steps is necessary. One sees that the predictions for this simple chaotic model are very good

plots for the time series $\hat{x}_{i,} \hat{y}_{j}$ will be identical for that of the pair $\hat{x}_{i}, \hat{y}_{j}$ if

$$
\begin{aligned}
& \Theta\left(\varepsilon^{x}-\left\|x_{i}-x_{j}\right\|\right) \Theta\left(\varepsilon^{y}-\left\|y_{i}-y_{j}\right\|\right) \\
& =\Theta\left(\varepsilon^{x}-\left\|\hat{x}_{i}-\hat{x}_{j}\right\|\right) \Theta\left(\varepsilon^{y}-\left\|\hat{y}_{i}-\hat{y}_{j}\right\|\right) \text { for } i, j=1, \mathrm{~N}
\end{aligned}
$$

Again, in general this condition will be difficult to satisfy. As an indication of the differences that may arise, Fig. 10 presents cross recurrence plots for two quasi-periodic signals. Figure $10 \mathrm{a}$ is for these two signals with no linear trend whilst Fig. 10b is for the same two signals with a linear trend added to the first. One can see that the two plots are quite different. In some instances, however, the removal of a linear trend makes quite small differences as we will see for the KAIK_e signal later in the paper.

\section{Analysis of GNSS data}

Nature of the data

Time series for crustal displacements using satellite acquired data are collected by the GeoNet organisation in
New Zealand, a collaboration between the NZ Earthquake Commission and GNS Science, using GNSS (popularly known as GPS) receivers and antennae. In order to explore the application of nonlinear time analysis to GNSS data, we have selected five of the operating GNSS stations shown as yellow triangles in Fig. 11a. At each station, three output files are available containing displacement records relative to a reference datum (Hofmann-Wellenhof et al. 2008) defined by the International Terrestrial Reference Frame (ITRF2008) for East, North and vertical displacements evaluated on a daily basis from data collected every second. The $1 \mathrm{~s}$ data contain noise from known and unknown sources and may be influenced by both deterministic and observational noise produced by the processes of data collection (Hofmann-Wellenhof et al. 2008). In these procedures, linear combinations of various signal frequencies are combined as a method of smoothing the $1 \mathrm{~s}$ data (Hofmann-Wellenhof et al. 2008). In addition, the $1 \mathrm{~s}$ data are aggregated from $1 \mathrm{~s}$ to 1 day time series. We have retained the raw supplied data, expressed as daily displacements with no further processing, since in any nonlinear analysis, it is never clear initially what is noise from measurement or other external sources (observational noise) and how much of the signal is 


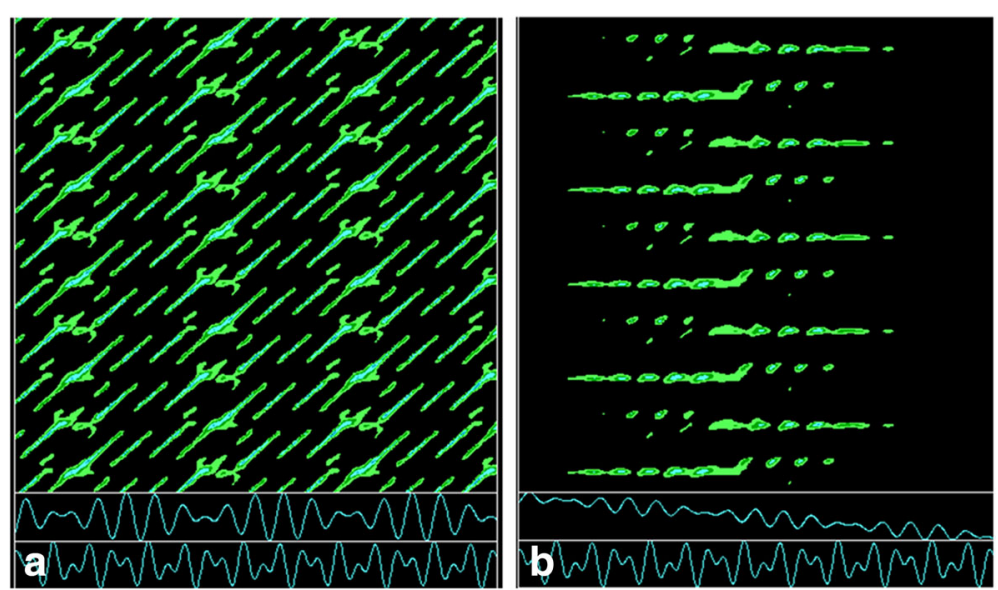

Fig. 10 The influence of a linear trend on a cross recurrence plot. a Cross recurrence plot for $y=\sin (x)+\sin (11 x / 9)($ top frame $)$ against $y=\cos (x)$ $+\sin (5 x / 3)$ (bottom frame). $\mathbf{b}$ Cross recurrence plot of $y=\sin (x)+\sin (11 x / 9)-0.1 x$ (top frame) against $y=\cos (x)+\sin (5 x / 3)($ bottom frame)

intrinsic to the nonlinearity of the dynamics (dynamical noise). It would be interesting to begin with the $1 \mathrm{~s}$ data in future work. An initial exploration, as this study is, should retain as much of the data as possible with a view to identifying externally induced (non-deterministic) noise at a later stage. A brief look at noise in these signals is given later in the paper. Some of the possible linear methods of noise reduction are considered in Goudarzi et al. (2012). At each station, data are collected for the easterly (suffix:_e), northerly (suffix:_n) and vertical (suffix:_u) displacements. In Fig. 11, we show only the easterly component data except for the stations CNST and PAWA where we explore the vertical component data. We also explore possible synchronisation between nearby stations using cross- and joint-recurrence plots. Since there is some interest in understanding synchronisation between two stations on the North Island of New Zealand with an event (marked as a red triangle in Fig. 11a) on the South Island (Wallace et al. 2017), we also explore synchronisation between two distant stations.

The raw data used here and reported at 1 day intervals contains relatively small gaps (about 6 days at most in the signals we investigated) that presumably arise from station down-time. We have retained these gaps for most analyses but have explored the effect of removing them. Such a process seems to make little difference to the details of both recurrence and cross recurrence plots but clearly is important if one wants to match events in cross and joint recurrence plots. Future work should explore nonlinear prediction methods in filling these gaps.

The emphasis in the use of GNSS time series for geotectonic purposes in most published literature is to establish the velocity imposed on the crust by plate tectonic processes. As such the data are processed
(Beavan and Haines 2001; Wallace et al. 2004) in order to arrive at a velocity field that is smooth and continuous over substantial parts of the surface of the Earth. From such studies, important constraints can be placed on that part of the deformation of the crust that is commonly referred to as the rigid body motions (Wallace et al. 2004, 2010). Many studies propose that the crust is made of microplates that may have slightly different rigid body motions (Thatcher 1995, 2007; Chen et al. 2004; Wallace et al. 2004, 2010) and although some may offer more continuous models (Zhang et al. 2004) the case for such micro-plates existing in New Zealand seems to be well established (Wallace et al. 2004, 2010). The deformation within such microplates is commonly thought of as elastic (McCaffrey 2002; Wallace et al. 2010) and such an assumption is reasonable if one is seeking a smooth, continuous distribution of velocities on the scale of the microplate. However, in this paper, we seek to understand something of the system dynamics of crustal deformation processes by examining the history of deformation, continuous and discontinuous, within these microplates together with the coupling between these microplates over time. As such, the rigid plate tectonic motions are, in a sense, noise as far as the signal is concerned whereas for geotectonic purposes the details of the signal, which are our interest, are noise that is commonly removed by intensive processing (Wallace et al. 2010).

The rigid body motions of the crust arising from plate tectonic motions constitute a vector field on the surface of the Earth whereas the history of displacements within a microplate can be represented as an attractor that describes the dynamics in phase space. In principle, the characteristics of the attractor should not be altered by the subtraction of rigid body velocities but there is an issue in defining how much of an observed trend in a 

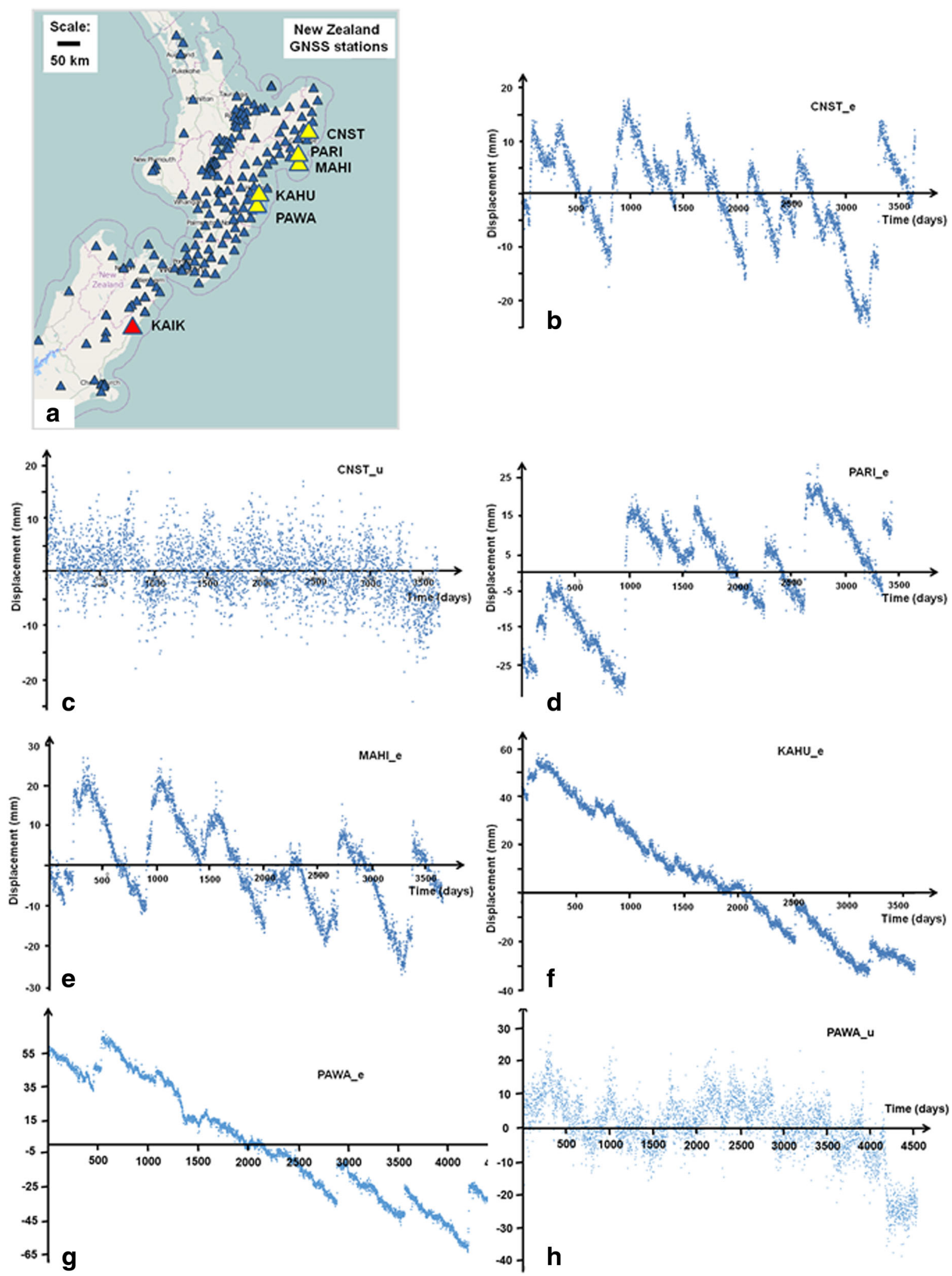

Fig. 11 Raw daily data for components of displacement from five GNSS stations on the North Island of New Zealand. a Locality map. The five stations are shown as yellow stars. The red star is station KAIK on the South Island that is examined for synchronicity with stations CNST and PAWA on the North Island later in the paper. b CNST_e. c CNST_u. d PARI_e. e MAHI_e. f KAHU_e. g PAWA_e. h PAWA_u 
GNSS signal arises from a rigid body motion is a contribution from regional plate tectonic motions and how much arises from elastic deformation or even from other internal permanent plastic/viscous deformation of the microplate. This is particularly the case if the overall trend is not linear.

For the recurrence plots presented here, we have elected not to remove the overall trend since the RQA measures for such plots are influenced by the trend although the trend, if pronounced, is clear in the plot. This is particularly true for KAHU_e and PAWA_e (Fig. 11e, f, but for other plots, the influence of the trend is minimal. We have removed the trend from the KAIK_e plot when we examine synchronisation between stations but recognise that such removal may have an influence on the apparent dynamics of the system; we examine the influence of such trend removal later in the paper.

It would seem from a cursory examination of many of the GNSS records from the North and South Islands of New Zealand that New Zealand is composed of an inter-locking mosaic of blocks and within each block the history of GNSS displacements have a similar history. It appears that each block moves whilst maintaining deformation compatibility at the boundaries of these blocks by combinations of boundary slip, block rotation and internal elastic, brittle and plastic deformation. There is evidence (Wallace et al. 2017 and this paper) that the motions of individual blocks are synchronised with others over quite large distances. The question arises therefore: How much of the average trend is to be attributed to the overall plate tectonic motions and how much is to be attributed to the nonlinear dynamics of the microplate? Although such a question is fundamental and is in need of detailed examination we elect to side-step the issue and unless indicated otherwise treat the raw data as an input to analyses.

\section{Recurrence analysis of GNSS data}

We begin by analysing the data from one station (CNST) in some detail to illustrate the procedures spelt out in Fig. 2 and then proceed to examine the other four other stations shown as yellow triangles in Fig. 11a in less detail. We then proceed to examine nonlinear synchronisation of displacement histories between stations CNST and PAWA, a distance of $\approx 220 \mathrm{~km}$, and between stations CNST, PAWA and KAIK, a distance of $\approx 440$ to $650 \mathrm{~km}$.

In all the recurrence/cross-recurrence/joint-recurrence plots for GNSS data, the embedding dimension is 10 and the time delay is 5 . The scaling is maximum distance, and the radius is 20\% (Webber and Zbilut 2005). The parameter $c$ is 5 so that four levels of contours appear in each plot. The signal for the raw data together with the time scale is shown at the base of each recurrence plot at the same linear scale as the plot. Cross reference to Fig. 11 gives finer detail of the absolute time scale for each plot.

Figure 12a, b shows the recurrence plots and associated signals for the raw daily data for CNST_e and CNST_u. We have not analysed CNST_n data. The contrast in appearance between Fig. 12a, b reflects the nature of the two signals. The CNST_e data comprise a number of discontinuities with downward non-stationary trends between discontinuities. This is represented on the recurrence plot by abrupt gaps in recurrence (black areas) with fading to the upper right patterns between gaps. The large black areas (up to $\approx 300$ days wide) where no recurrences occur are particularly evident immediately prior to large discontinuities in the displacement record.

The recurrence plot for CNST_u (Fig. 12b) is much more highly populated with recurrences. Regions of no recurrence (black) tend to occur immediately prior to changes in the patterns in the raw data but these gaps are $\approx 50$ days wide as opposed to up to a year in the CNST_e recurrence plot.

The essential attributes of the CNST_e recurrence plot are expressed in the RQA analysis of Table 3. The relatively low level of \%REC is expressed by the high proportion of black areas in Fig. 12a. The signal is highly deterministic as indicated by the high values of \%DET. DMAX is large which indicates a small value for the first Lyapunov exponent; this in turn indicates the potential for good predictability. The entropy $(\mathrm{ENT}=4.4 \mathrm{bits} / \mathrm{bin})$ is larger than that of the Hénon system $(E N T=2.56$ bits/bin) and so suggests predictability may be more difficult for CNST signals than for the Hénon system.

These values of RQA measures for CNST_e are to be contrasted with those for CNST_u which reflects the more diffuse nature of the latter signal. In particular, the first Lyapunov exponent indicates that predictability may be difficult.

Similar observations to the above hold for the other signals examined: large gaps (black areas) in recurrence tend to occur prior to large discontinuities in displacement, determinism is high and the first Lyapunov exponent is small. Obvious differences in "_e recurrence plots exist for data sets that show significant non-stationarity: recurrence tends to be restricted to a relatively narrow zone either side of the main diagonal LOI but again discontinuities in the signal are preceded on the recurrence plots by gaps in recurrence.

As a final way of analysing recurrence plots, we show in Fig. $12 \mathrm{~h}$ a series of windows along the main identity diagonal. Within each window, a different pattern of recurrence exists that reflects the details of the signal. One can undertake an RQA within each window and map the way in which the RQA measures evolve with time. This is done in Figs. 16 and 19. The procedure 

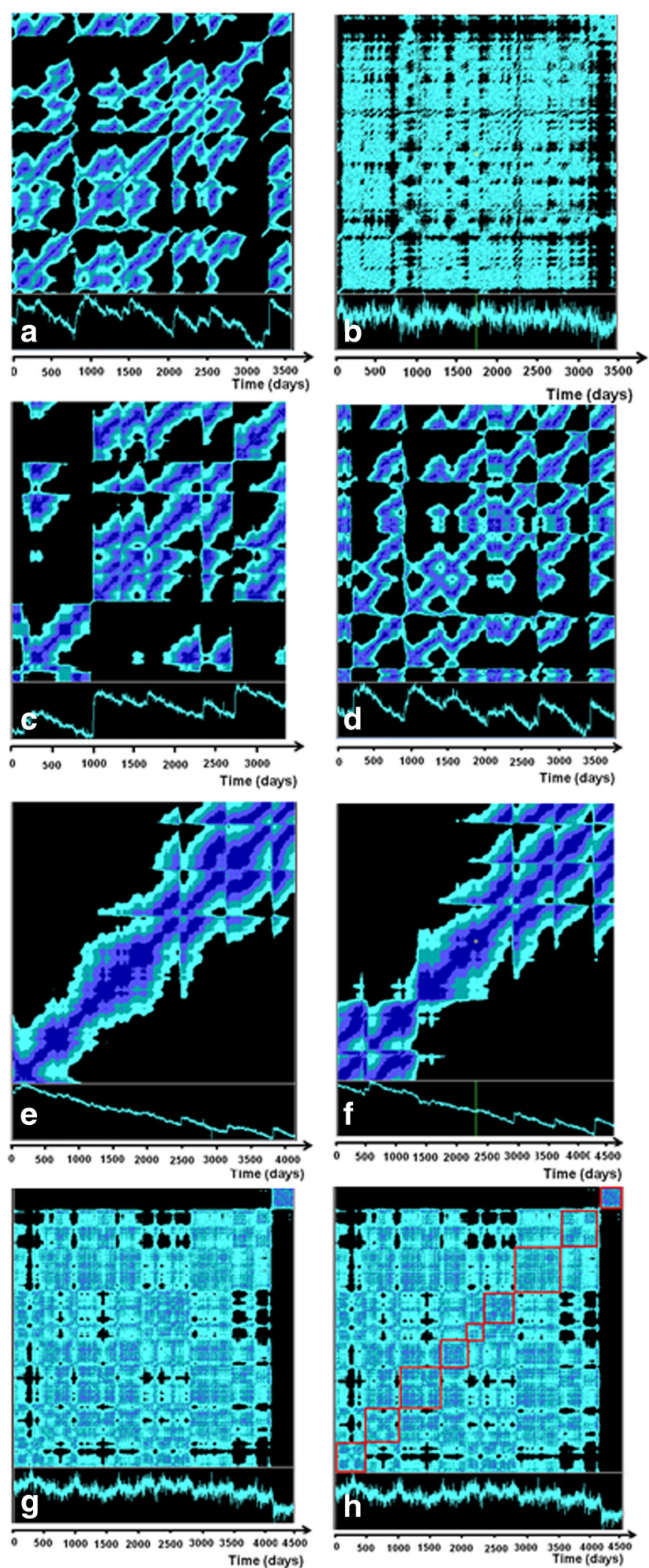

Fig. 12 (See legend on next page.) 
(See figure on previous page.)

Fig. 12 Recurrence plots for stations marked as yellow stars in Fig. 11a. In all figures the embedding dimension is 10 and the time delay is 5 . The scaling is maximum distance and the radius is 20\% (Webber and Zbilut 2005). The parameter $c$ is 5 so that 4 levels of contors appear in each plot. The signal for the raw data is shown at the base of each recurrence plot at the same linear scale as the plot. Details of these signals together with the time scale are shown in Fig. 11. a CNST_e. b CNST_u. c PARI_e. d MAHI_e. e KAHU_e.f PAWA_e. g PAWA_u. h PAWA_u with sliding windows marked in red. Within each window a different pattern of recurrence occurs. The zero for the time scale in each of these plots and in subsequent plots in this paper is the zero for the relevant time scale in Fig. 11

is known as a sliding window analysis (Webber and Zbilut 2005).

\section{Embedding dimension and the nature of the attractor}

In Fig. 13, we show plots of the correlation dimension, given by (3), and the percentage of false nearest neighbours against embedding dimension for CNST_e (Fig. 13a, b) and CNST_u (Fig. 13c, d). The correlation dimension plot tends to deviate from a straight line somewhere in the range 8 to 10 indicating a relatively high value for the embedding dimension. This indicates that the underlying dynamics of the system involve 8 to 10 state variables; certainly more state variables are involved than in the Lorentz system (5). The percentage of false nearest neighbours gives little useful information as far as the embedding dimension is concerned and continues to decrease as the embedding dimension increases. This again indicates that the attractor is complicated and that it is difficult to view the attractor in its true embedding dimension with the data available. More data are needed to cover the attractor and sample all possible states.

In Fig. 14, we show a projection of the attractor for the CNST_e data set shown in Fig. 11b. As indicated earlier, the embedding dimension for this attractor is probably about 10 so Fig. 14 is a projection from this 10-dimensional state space into three dimensions. The fact that many trajectories cross each other in this projection is an indication of the large number of false neighbours in three dimensions. Also note the presence of knot-like "outliers" in the system that are visited rarely but have a complicated shape with false neighbours in three dimensions. This indicates that the local estimates of the embedding dimension can be quite variable (Small 2005) and we tentatively assume that these complications in the attractor are responsible for any difficulties involved in using false nearest neighbours as a means of estimating the embedding dimension.

\section{Noise reduction}

As an example of the differences between nonlinear and linear noise reduction procedures, we first present in Fig. 15 two examples of the nonlinear noise reduction procedure discussed earlier in the paper. Here, the embedding dimension is taken as 10 and the delay, 5 . Figure $15 \mathrm{a}$ shows approximately $76 \%$ noise reduction and the corresponding plot of correlation dimension against embedding dimension is shown in Fig. 15b. This shows a reduction in the possible embedding dimension to about 5. In Fig. 15c, 80\% noise reduction is shown for CNST_u and the corresponding plot of correlation dimension against embedding dimension is shown in Fig. 15d with a slight reduction in the indicated

Table 3 RQA measures for selected GNSS data sets on the North Island of New Zealand

\begin{tabular}{|c|c|c|c|c|c|c|c|c|c|}
\hline Station & Data set & \%REC & \%DET & DMAX & ENT & TREND & \%LAM & VMAX & TTIME \\
\hline CNST & $\mathrm{e}$ & 39.49 & 97.88 & 3588 & 4.4 & -6 & 98.5 & 837 & 51.56 \\
\hline $\mathrm{CNST}^{*}$ & e_trunc & 38.44 & 97.48 & 2687 & 4.28 & -7.05 & 98.21 & 411 & 40.1 \\
\hline CNST** & e_trunc_n & 36.44 & 97.48 & 2688 & 4.28 & -7.02 & 98.21 & 411 & 40.11 \\
\hline CNST ${ }^{* * *}$ & e_reg_n & 36.48 & 97.31 & 2676 & 3.8 & -10.02 & 97.99 & 446 & 31.85 \\
\hline CNST & u & 6.9 & 29.13 & 45 & 0.89 & -1.92 & 45.88 & 42 & 2.59 \\
\hline CNST ${ }^{* * * *}$ & $u_{-} n$ & 27.77 & 65.81 & 114 & 1.6 & -6.95 & 76.54 & 154 & 3.58 \\
\hline PARI & e & 39.26 & 98.73 & 3380 & 4.5 & -13.77 & 99.07 & 898 & 70.36 \\
\hline MAHI & e & 42.38 & 98.26 & 3685 & 4.38 & -9.77 & 98.79 & 841 & 60.7 \\
\hline KAHU & e & 37.47 & 99.47 & 4183 & 4.51 & -27.64 & 99.62 & 1315 & 181.64 \\
\hline PAWA & e & 38.16 & 99.50 & 4526 & 5.14 & -25.24 & 99.65 & 1326 & 200.18 \\
\hline PAWA & u & 56.32 & 95.54 & 1796 & 2.92 & -15.05 & 96.76 & 1027 & 18.05 \\
\hline
\end{tabular}

${ }^{*}$ CNST_e data truncated from 3650 days to 3300 days

**Truncated CNST_e data with nonlinear noise removal

***Truncated CNST_e data with regional filter method noise removal (Beavan et al. 2004)

****CNST_u data with nonlinear noise removal 


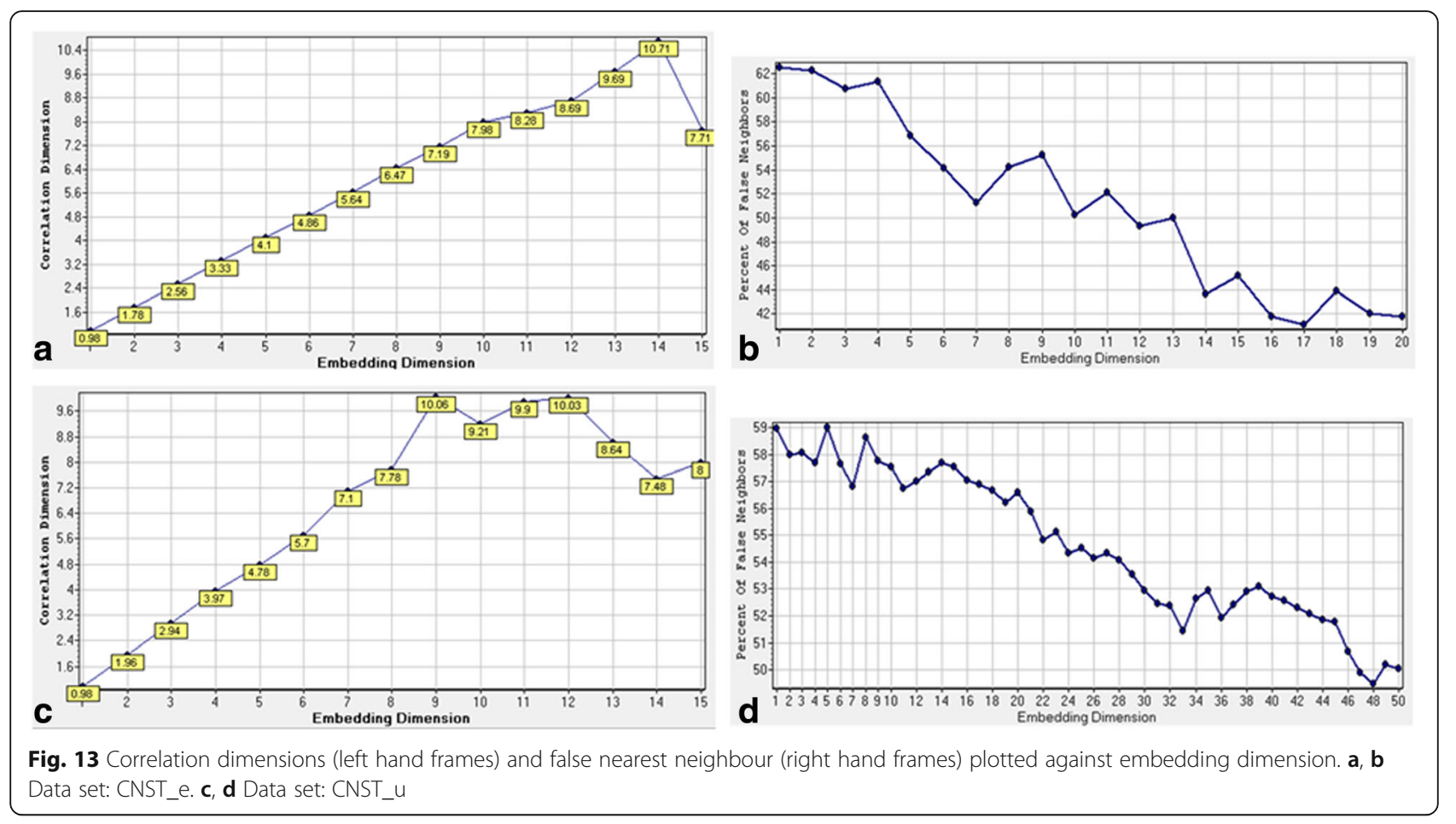
Data set: CNST_e. c, d Data set: CNST_u

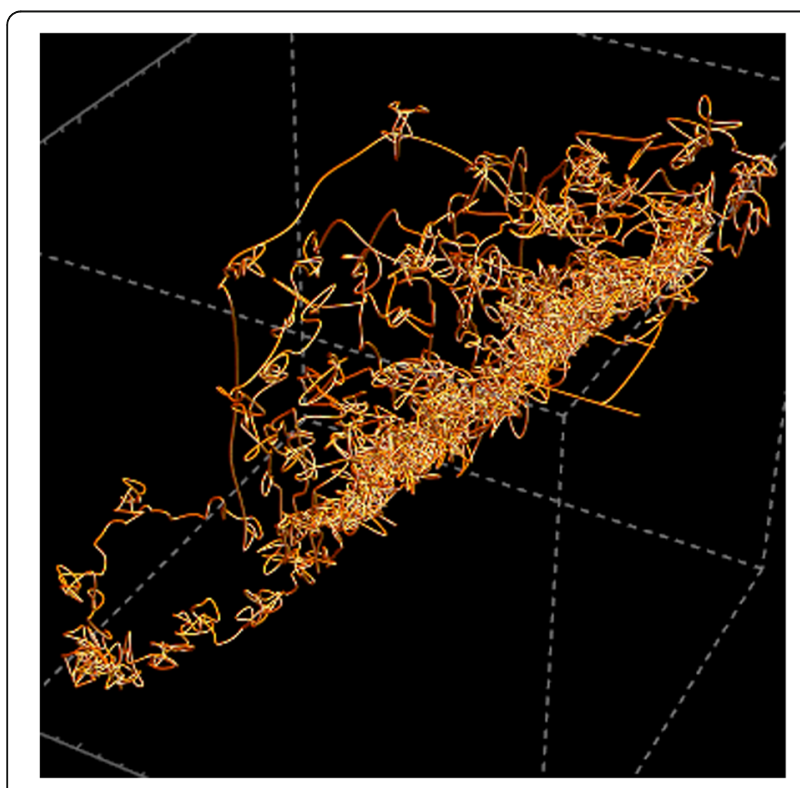

Fig. 14 Projection of the attractor for CNST_e data from a higher dimension (perhaps an embedding dimension of 10) into three dimensions. The attractor has been constructed using a time delay of 30 days according to the method discussed in the "The invariant measures" section. See Hobbs and Ord (2015), pp. 227-228 for details of the construction method embedding dimension compared to Fig. 13c. An attempt was made to optimise the noise reduction in these two cases by exploring different embedding dimensions and delays.

In Table 4, the RQA data are shown for CNST_e (raw data, column 1), CNST_e with nonlinear noise reduction (column 2) and CNST_e with noise removed by the linear regional filter described by Beavan et al. (2004); this latter method of noise removal has now been discontinued by GeoNet. The nonlinear noise removal process leads to no or insignificant changes in the RQA measures whereas the regional filter method produces $5 \%$ increase in \%recurrence, an $11 \%$ increase in entropy, a $42 \%$ decrease in trend and a $21 \%$ increase in trapping time. Thus, although the data may appear "smoother", the basic measures of the dynamics of the system have been significantly altered by the linear filtering method. A basic premise is that any data reduction method should preserve dynamic noise and it seems that the linear method has removed some such noise in this example.

\section{Sliding window analysis of CNST_e data}

A useful way of analysing recurrence plots is called the sliding window method (Marwan et al. 2007a) whereby the recurrence plot is divided into small windows along the main diagonal LOI which may or may not overlap as desired (Fig. 12h). RQA measures are produced in each window enabling plots of these measures to be made through the history of the time series. In Fig. 16, 


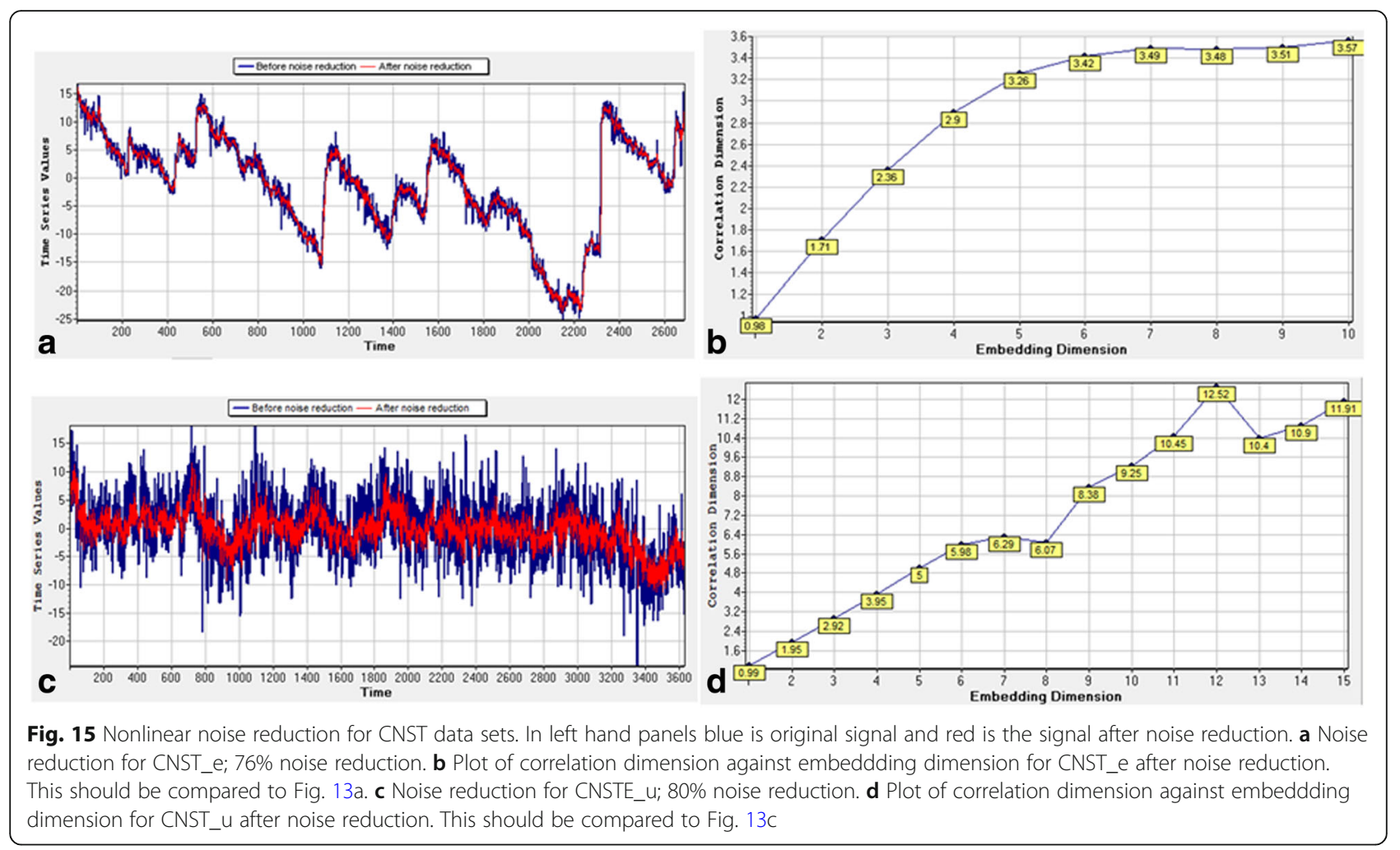

such an analysis is shown for CNST_e data. This enables the RQA data to be compared directly with local patterns in the raw data. In particular, we are interested in RQA measures that may serve as precursors to the main discontinuities in the displacement record.

In this analysis, the window size is 100 days and there is a 50 day overlap between windows so that each window can "see" 100 days ahead; this represents two black dots on the RQA signals shown in Fig. 16. Each dot is plotted at the beginning of the 100 day window so each dot represents the RQA measure of the signal 100 days ahead. Here, we concentrate on the main displacement discontinuity that begins about day 3307 and continues until about day 3320. One can see that the mean and standard deviation track the signal precisely and so are of little use as precursors. However, the \%LAM and DMAX measures both behave anomalously 100 days before the displacement discontinuity at $\sim 3307$ days and so are candidates as precursors for this event although it is appreciated that this event is not sharp and extends in a compound manner starting at $\approx 3200$ days; others are possible but higher resolution (say 0.1 day binning) is necessary before one can be definitive.

\section{Nonlinear prediction of CNST_e data}

A test of whether one has a reasonable data model is to attempt some form of nonlinear prediction. We have attempted this for the CNST_e data set using the first

Table 4 Comparison of RQA measures for data set CNST_e, as raw data (column 1), with nonlinear noise reduction (column 2) and with noise reduction using the regional filter method (column 3; Beavan et al. 2004)

\begin{tabular}{llllll}
\hline Data set & $\begin{array}{l}\text { 1. CNST_e } \\
\text { Raw one-day data }\end{array}$ & $\begin{array}{l}\text { 2. CNST_e } \\
\text { Nonlinear noise removal }\end{array}$ & $\begin{array}{l}\text { 3. CNST_e } \\
\text { Regional filter }\end{array}$ & \% change of \# 2 with respect to 1 & \% change of \# 3 with respect to 1 \\
\hline \%REC & 38.44 & 38.44 & 36.48 & 0 & 5.10 \\
\%DET & 97.48 & 97.48 & 97.31 & 0 & 0.17 \\
LMAX & 2687 & 2688 & 2676 & -0.04 & 0.41 \\
ENT & 4.28 & 4.28 & 3.8 & 0 & 11.21 \\
TREND & -7.05 & -7.02 & -10.02 & -0.43 & -42.13 \\
\%LAM & 98.21 & 98.21 & 97.99 & 0 & 0.22 \\
VMAX & 411 & 411 & 446 & 0 & -8.52 \\
TTIME & 40.1 & 40.1 & 31.85 & 0 & 20.57 \\
\hline
\end{tabular}



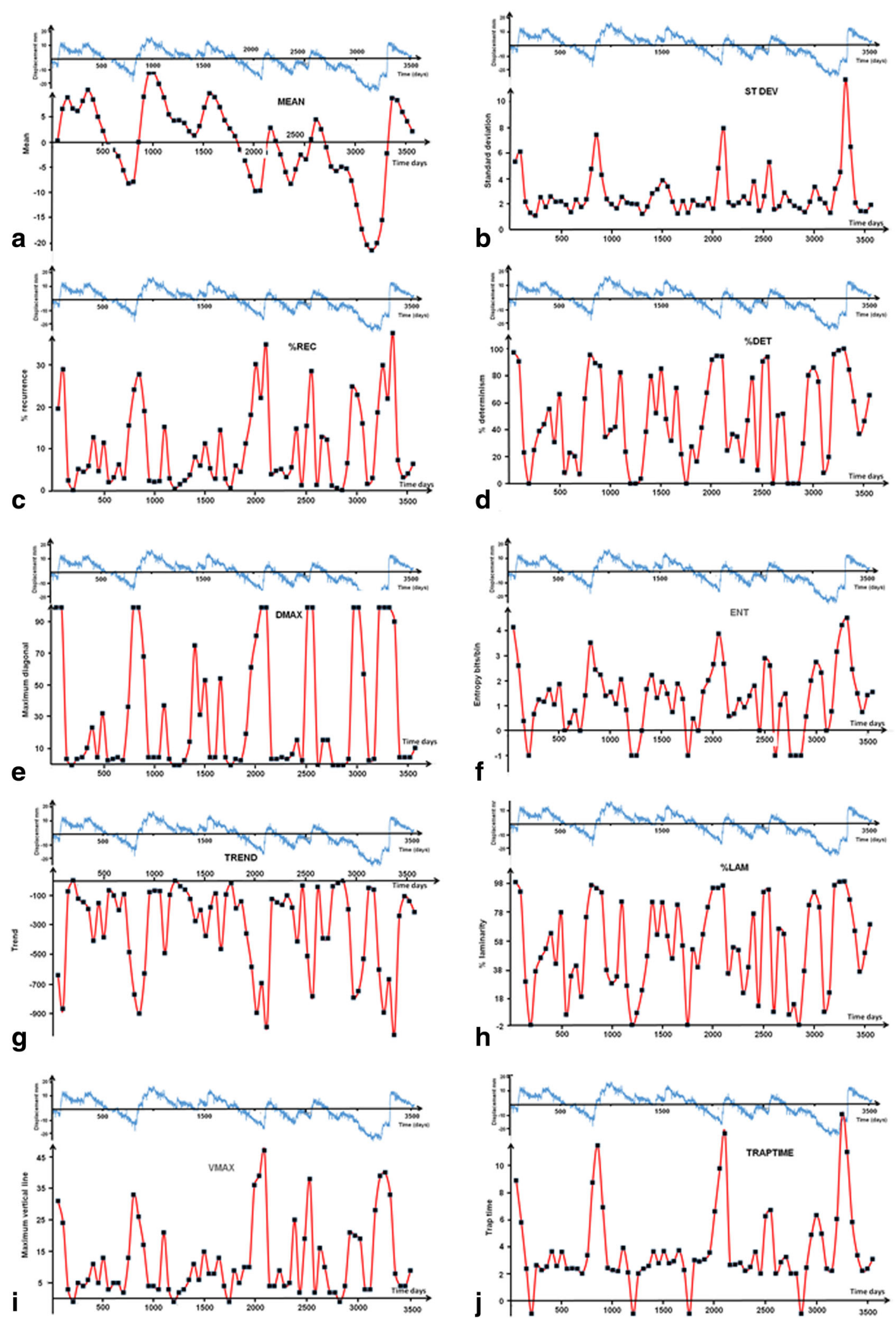

Fig. 16 RQA measures for a sliding window of width 100 days and an overlap beween windows of 50 days for the data set CNST_e shown at the top of each frame. a Mean. $\mathbf{b}$ Standard deviation. c \%REC. $\mathbf{d} \% D E T$. e DMAX. f ENT. g TREND. $\mathbf{h} \% L A M . \mathbf{i}$ VMAX. $\mathbf{j} T T I M E$ 
3200 days of the signal as a training set and an attempted prediction for the period 3201 to 3501 days. The results are shown in Fig. 17 which represents the 300 days starting at day 3201 and ending at day 3500 for an embedding dimension of 10 , a delay of 10 , a local linear weight predictor and a Gaussian kernel. The actual values are plotted in blue and the prediction in red. The normalised error (in green) starts high at $\approx 1.4$ remains high and drops to below 1 at the main jump in displacement. The final normalised error is $\sim 0.98$ but is still marginally better than linear predictors based on (7). The predicted signal hugs the details of the observed data quite nicely including the large displacement at about 105 days. This analysis again confirms that the embedding dimension of the attractor is relatively large. Reducing the embedding dimension below 10 or increasing the embedding dimension to 12 give normalised errors far greater than 1 .

\section{Synchronisation of data sets}

It is clear that some of the large displacement events in New Zealand occur at near to the same time. Thus the same large displacement events can be observed synchronously at several stations (Wallace et al. 2017, Fig. 2). This represents synchronisation of large displacements over distances of at least $220 \mathrm{~km}$. Recently, Wallace et al. (2017) have proposed that the magnitude 7.8 seismic Kaikōura event triggered large displacement events 250 to $600 \mathrm{~km}$ away on the North Island for 1 to 2 weeks after the South Island event. Whilst such synchronisation is clear, it is of interest to see if more subtle forms of synchronisation exist and, if so, over what length and time scales does synchronisation occur? Also an understanding of where such synchronisation sits in the classification of synchronisation types described earlier in the paper and details of the frequencies at which synchronisation occurs would shed light on the dynamics of crustal deformation. In what follows, we employ cross-recurrence and joint recurrence plots to detect synchronisation between stations, to clarify details of the synchronisation and to classify the mode of synchronisation.

\section{Synchronisation between stations on the North Island}

In Fig. 18, we show synchronisation between signals for CNST_e and PAWA_e. Figure 18a is a cross recurrence plot and shows that gaps in synchronisation between the two signals (black areas on the cross recurrence plot marked by white arrows) begin months before major displacement events at both CNST and PAWA, and synchronisation begins again immediately after an event. The ratio of recurrence time scales for the two stations, $T_{\mathrm{CNST}} / T_{\mathrm{PAWA}}$, is $\approx 3: 5$ as shown by the slope of the LOSs and using (5). There are places in Fig. 18a just before large displacement events where the LOS is almost horizontal indicating that $T_{\mathrm{CNST}} / T_{\mathrm{PAWA}}$ switches from $\approx 3: 5$ to a large number $\left(\beta\right.$ in (5) approaches $0^{\circ}$ as $T_{\mathrm{CNST}} / T_{\mathrm{PAWA}} \rightarrow \infty$ ). These places (marked with red arrows) of low $T_{\mathrm{CNST}} / T_{\mathrm{PAWA}}$ correspond to discontinuities in the CNST_e displacement plot. Discontinuities in the PAWA_e displacement plot correspond to discontinuities in the LOSs with no change in $T_{\mathrm{CNST}} / T_{\mathrm{PAWA}}$.

The joint recurrence plot is shown in Fig. $18 \mathrm{~b}$ and shows a high degree of joint recurrence along a single LOS for the early part of the history and widens out to have a higher proportion of joint recurrences as the major event is approached. After the major event, the joint recurrences are still strongly synchronised but the pattern of joint recurrences has broadened even further.

Figure 18c, d shows the probability of a recurrence versus frequency (1/lag in days) for CNST_e and PAWA_e respectively. Both signals have fractal distributions with respect to time lag for low frequencies but are more or less independent of frequency at high frequencies which is where the majority of recurrences occur and where the power in the signal exists. The details of the distributions are quite different for the two stations indicating that no simple phase or frequency locking exists between these

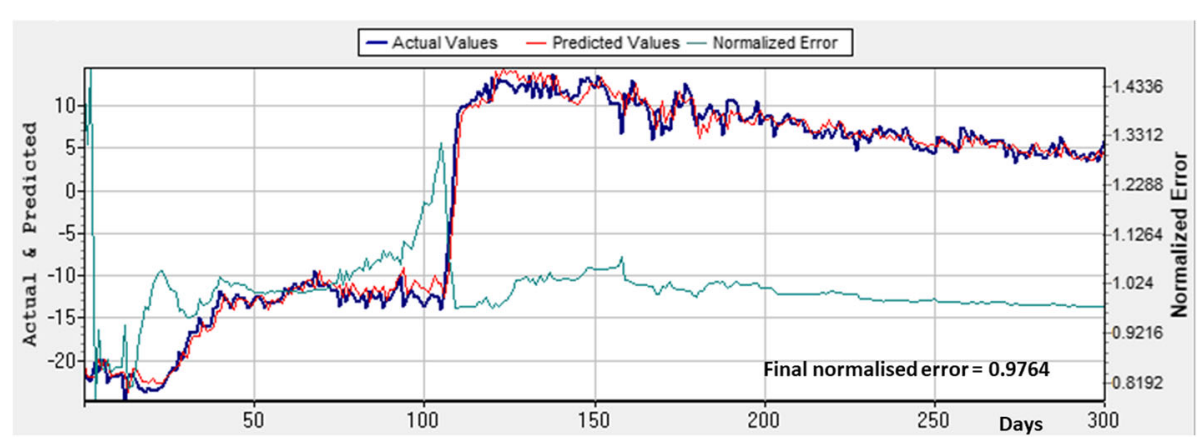

Fig. 17 Nonlinear prediction of CNST_e signal. The blue curve is the observed signal, the red is predicted and the green curve is the normalised error which is less that one and so marginally better than a linear prediction model given by (7). The prediction lags behind the observed signal by 1 day so is not ideal for practical predictive purposes but is a good test of the data model 


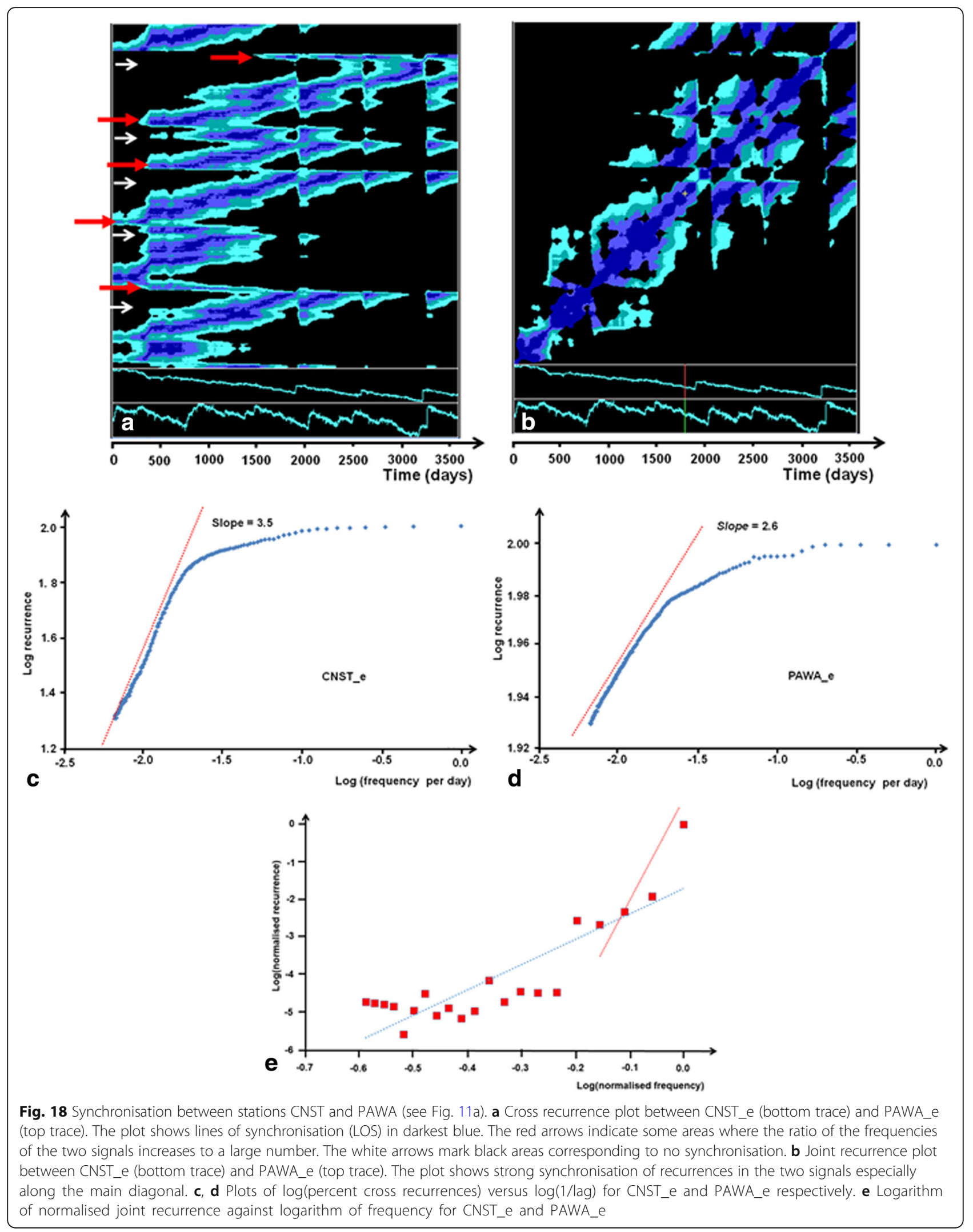


two stations. Figure 18e shows the scaling on a log-log plot of the probability of a joint recurrence against frequency (both normalised). The relation indicated by the dotted line is:

\section{normalised recurrence probability is proportional to ${\text { (normalised frequency })^{11.6}}$}

indicating that all of the power in the signal is partitioned into the highest frequencies. Since almost all (99\%) of the joint recurrences occur in the four or five highest frequencies in Fig. 18e, the partitioning of power into the highest frequencies is far more pronounced (as indicated by the dotted red line) than indicated by the above scaling relation. These observations indicate that the synchronisation between CNST and PAWA is a form of generalised synchronisation.

\section{Synchronisation between stations on the North Island and KAIK on the South Island}

The spectacular observations of Wallace et al. (2017) that the magnitude 7.8 Kaikoura seismic event in the South Island of New Zealand is followed for a few weeks by slow displacement events $250-600 \mathrm{~km}$ distant on the North Island indicates clear lag synchronisation over large distances. The questions we want to address are the following: do other forms of synchronisation exist over these large distances and, if so, what form do they take and is there information in the form of precursors in the synchronisation patterns? We first characterise the KAIK displacement history using a sliding window with RQA and then investigate cross recurrence plots between CNST and KAIK and between PAWA and KAIK.

Figure 19 shows the results of a sliding window analysis for the KAIK_e signal over a time period identical for signals from CNST and PAWA. The Kaikoura event occurs at day 3317 and so this event in Fig. 18 is in the 50 day window following the red star in each RQA plot. The window is 100 days wide and the overlap between windows is 50 days. This means that each black dot in Fig. 19 can "see" two black dots ahead. The RQA measures for the 100 day wide window are plotted at the day corresponding to the beginning of the window. Any precursors for KAIK_e events must therefore be evident two dots or more before the red star. A measure is deemed useful as a precursor to an event if the measure rises to more than twice the mean of the total signal for that measure for the period of 100 days before the event.

We see that the following RQA measures are not suitable as precursors to the Kaikoura event: mean, standard deviation, TTIME and TREND. The other RQA measures \%REC, \%DET, DMAX, ENT, \%LAM and VMAX seem to be useful precursors and increase to well over the mean of the measure 100 days before the Kaikoura event. These measures are connected to the determinism and the organisation of recurrence states and indicate that the processes operating in the system are becoming more organised for about 3 months at least before the Kaikoura event. The question then arises: do stations in the North Island "know" about this organisation process?

Figure 20 shows cross and joint recurrence plots for *_e time series between PAWA and KAIK. Figure 20a indicates strong synchronisation between PAWA and KAIK at punctuated intervals for a decade before the Kaikoura event. The ratio of time scales, $T_{\mathrm{PAWA}} / T_{\text {KAIK }}$, as indicated by the dark blue LOSs in Fig. 20a and from (5) is approximately 1:1 over large portions of the history but locally as in Fig. 20d the ratio increases to large values possibly $>20$ at places indicated by the red arrow. Figure 20c shows that the ratio has increased to $3-5$ before the main Kaikōura event. These relations presumably reflect a form of generalised synchronisation. Figure 20b shows synchronisation of joint recurrences over a narrow range of recurrences for the same period as is shown in Fig. 20a.

Figure 21 shows strong synchronisation between CNST and KAIK at punctuated intervals over a period of $\sim 3000$ days with $T_{\mathrm{CNST}} / T_{\mathrm{KAIK}}$ large but not easily quantified. If the ratio of the time scales is $\geq 11$ then the slope of the LOSs is $\geq 85^{\circ}$ so that the LOSs in Fig. 21 indicate that $\left(T_{\mathrm{CNST}} / T_{\mathrm{KAIK}}\right) \geq 11$ and probably closer to 20. Figure 21a stops the displacement time series 772 days before the Kaikoura event and shows that synchronisation is well established with relatively strong synchronisation beginning about 270 to 350 days before any large slow event and ending as that individual event ends. These same relations regarding the relations of synchronisation to the displacement history hold in Fig. 21b, c, d that extend the plot first to 72 days and then 2 days and 1 day before the Kaikōura event. Strong synchronisation is already apparent 72 days before the Kaikoura event and begins to drop off as the Kaikōura event approaches. These relations are clear when the plot is extended to just after the event (Fig. 21e) where the degree of synchronisation just before the Kaikoura event swamps the degree of synchronisation associated with displacement events earlier in the history.

We conclude that there is strong nonlinear generalised synchronisation between stations CNST and PAWA on the North Island with station KAIK on the South Island in a punctuated manner for a period of at least 9 months before the magnitude 7.8 Kaikōura event. For $\approx 270$ days before the Kaikoura event, the degree of synchronisation between CNST and KAIK intensifies dramatically and ceases at the Kaikōura event. However, similar patterns of synchronisation occur associated with every 

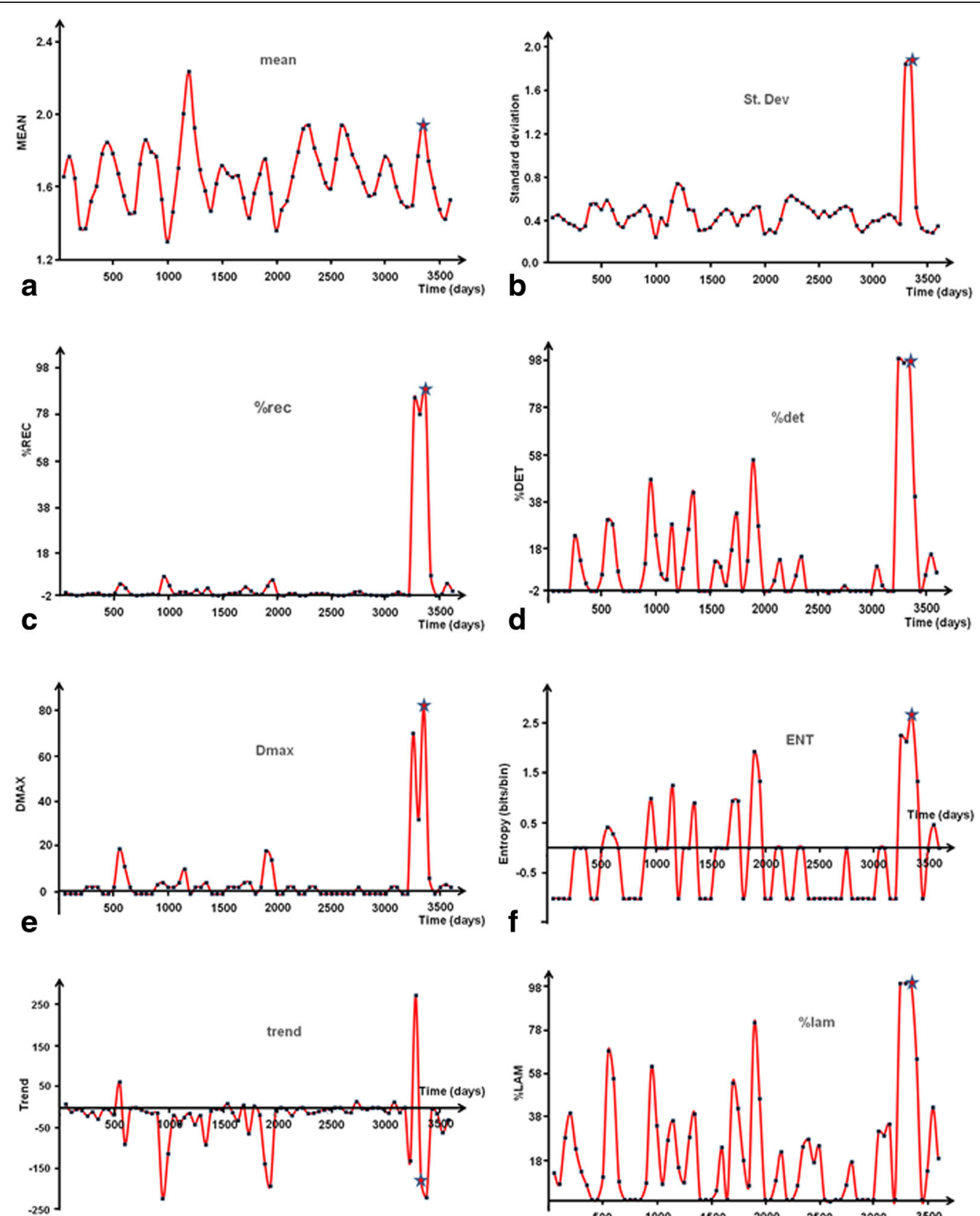

g
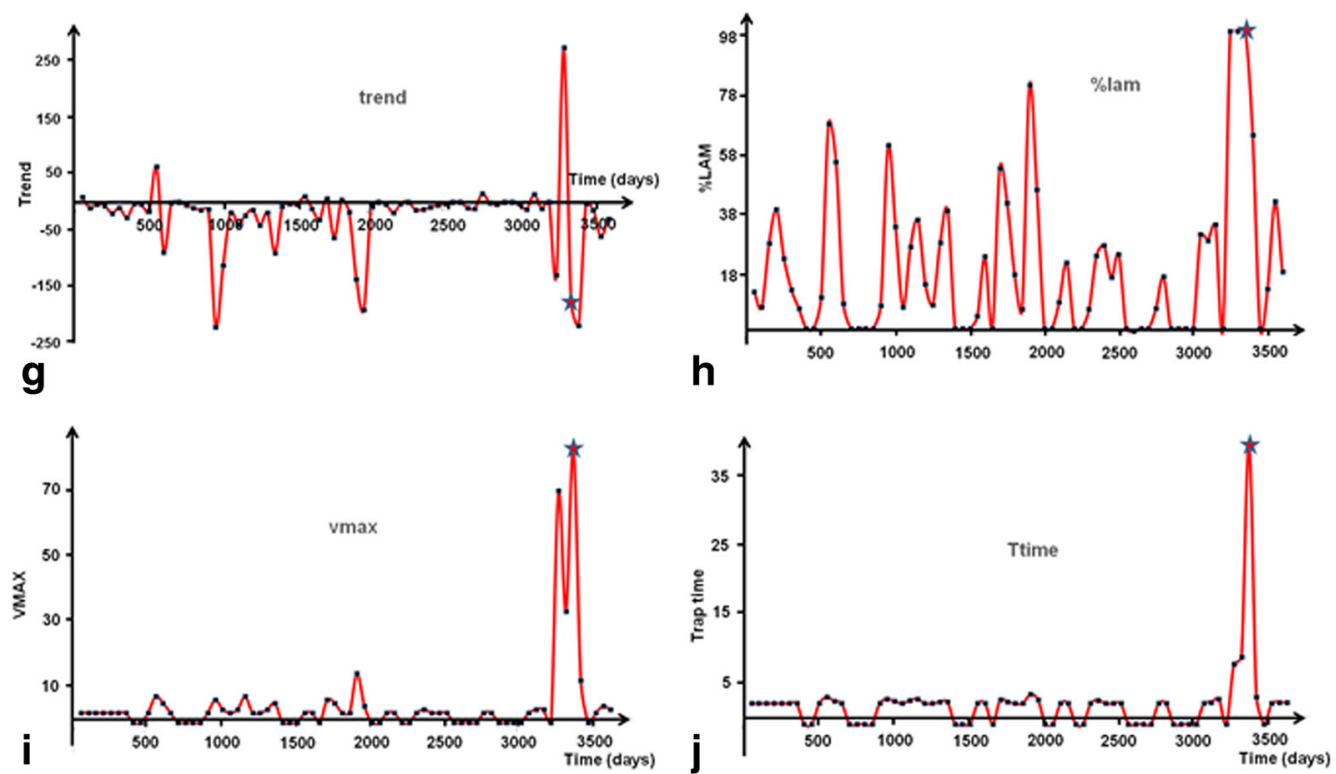

Fig. 19 (See legend on next page.) 
(See figure on previous page.)

Fig. 19 Sliding windows RQA for station KAIK. The sliding window is 100 days wide with an overlap between windows of 50 days. The main displacement event occcurs in the 50 day window following the red star. For an RQA measure to be a useful precursor it should depart by a factor of two or more from the mean of that measure 100 days (two black dots) before the main event. a Mean. b Standard deviation. c \%REC. d \%DET. e DMAX. f ENT. g TREND. $\mathbf{h} \% L A M . \mathbf{i} V M A X . \mathbf{j} T T I M E$

displacement event at CNST for the previous 3 years. These synchronisation events act as powerful precursors to both minor and major displacement events.

We attribute changes in $\beta$ in cross recurrence plots between two signals to changes in the frequency content of one or both signals. For instance, consider a situation where $\beta$ changes for $45^{\circ}$ to $90^{\circ}$ as occurs in Fig. 18 a, c. This can result from a change where the frequency contents of both signals are equal to a situation where the frequency content of one signal does not change but all the power in the other signal is partitioned into the highest frequency as occurs in Fig. 18e.
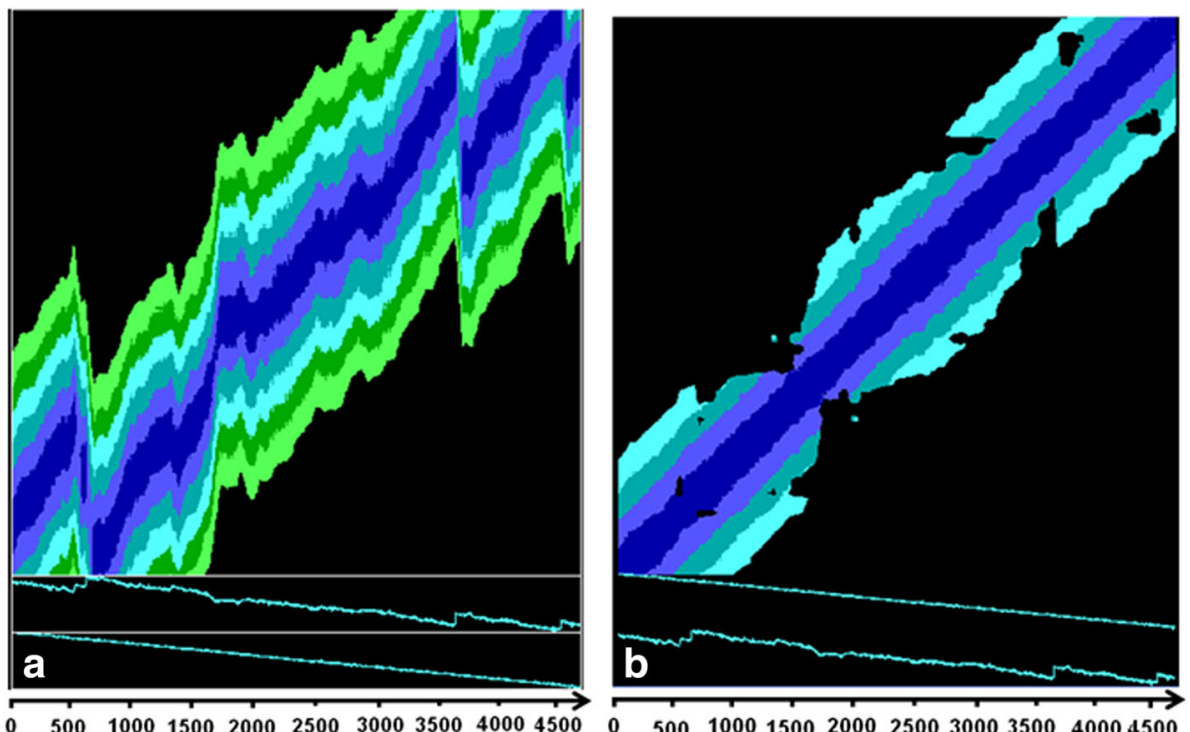

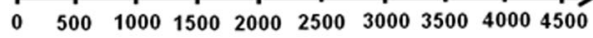

$0 \quad 500100015002000250030003500 \quad 40004500$

Time (days)

Time (days)
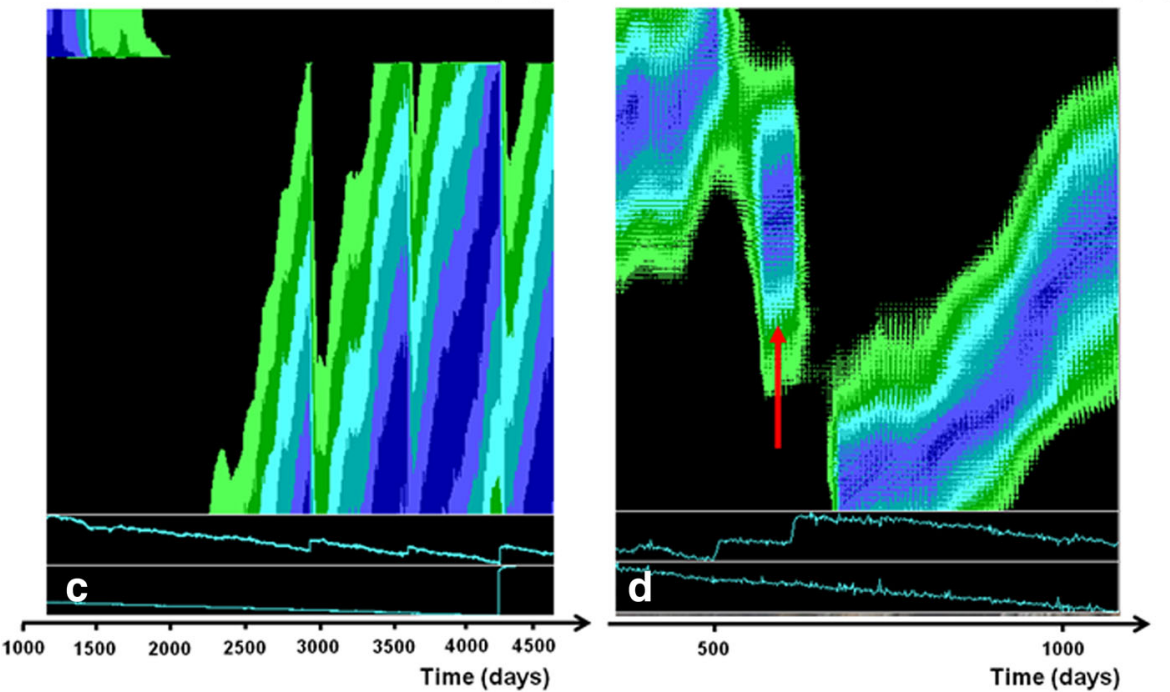

Fig. 20 Synchronisation between signals from station PAWA on the the North Island with KAIK signals on the South Island (see Fig. 11a). a Cross recurrence plot between PAWA_e and KAIK_e. PAWA_ upper frame, KAIK_e signal lower frame (b) Joint recurrence plot between PAWA_e and KAIK_e. KAIK_e signal upper frame, PAWA_e signal lower frame. c Cross recurrence plot between signals PAWA_e (top frame) and KAIK_e (lower frame) coving the period of the Kaikōura event. $\mathbf{d}$ Zoom into early part of Fig. 20a showing the steep LOS (red arrow) accompanying a major displacement jump 

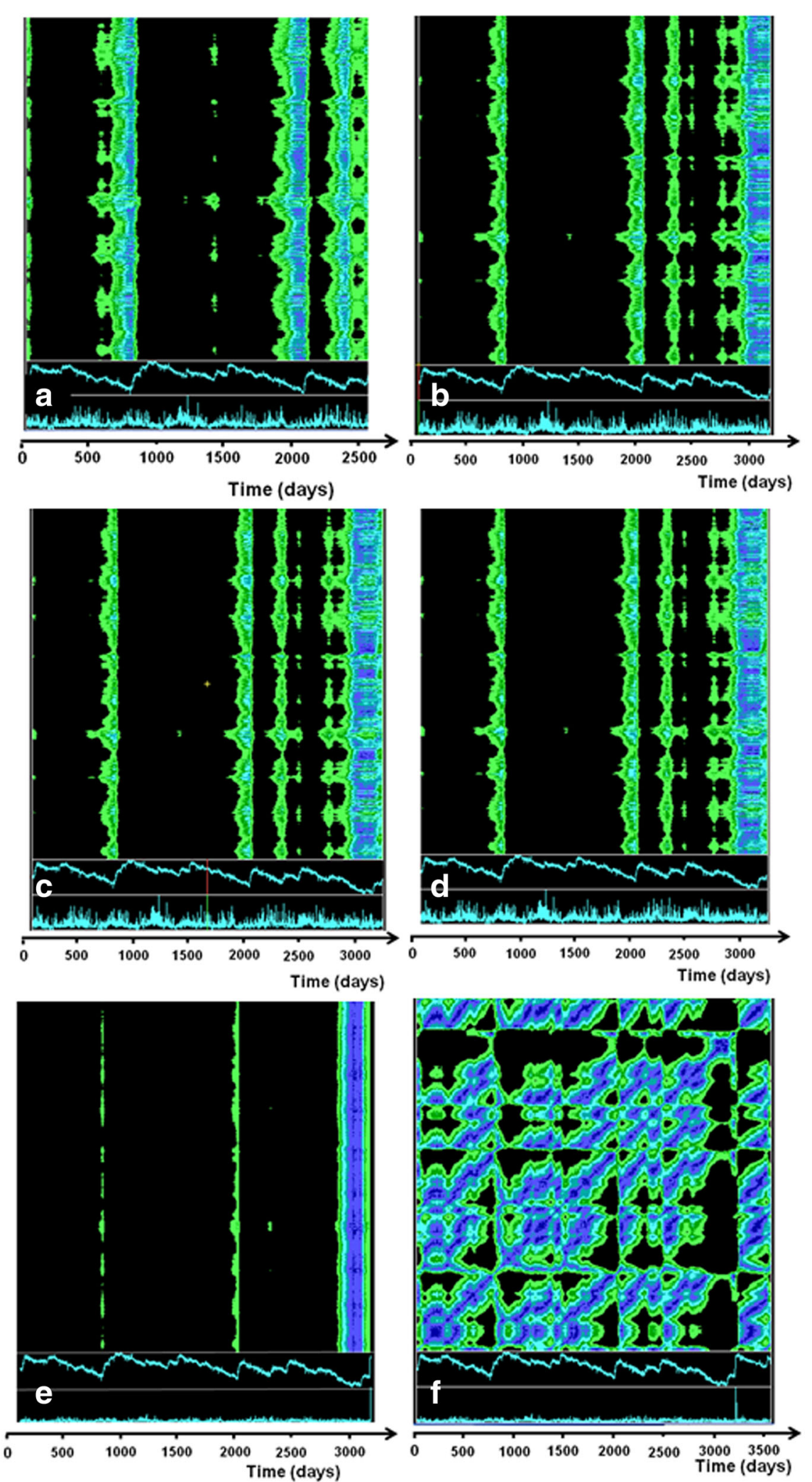

Fig. 21 (See legend on next page.) 
(See figure on previous page.)

Fig. 21 Cross recurrence plots and joint recurrence plot for CNST_e (top frame) and KAIK e (bottom frame: with downward trend removed) signals, (a) 772 days before the Kaiköura event. b 72 days before the Kaikōura event. c 2 days before the Kaiköura event (d) 1 day before the Kaikōura event, (e) 3 days after the Kaikōura event. The ratios of the characteristic frequencies for CNST_e and KAIK_e signals are large so that the LOSs are straight vertical lines. Figures $\mathbf{b}, \mathbf{c}$ and $\mathbf{d}$ appear almost identical but show the gradual increase in the presence of a low recurrence region on the right hand side of the plot as the Kaikōura event is approached. $\mathbf{f}$ Joint recurrence plot

We show sliding window joint recurrence quantification for CNST_e and KAIK_e (linear trend removed) in Fig. 22. Only \%REC (a), DMAX (b), $\operatorname{VMAX}(\mathrm{c})$ and TTIME $(\mathrm{d})$ are shown since they give the most unambiguous precursors to the Kaikōura event, which occurs in the 50 day window following the yellow star in each plot. As in previous sliding window analyses in this paper, the window is 100 days wide and there is an overlap of 50 days for successive windows. The result for each window is plotted at the start of the window. These measures show significant departures from the mean for each measure 100 days before the window containing the Kaikoura event indicating that these measures act as precursors over this time period.

The influence of including the linear trend for KAIK_e in the analysis is shown in Fig. 23 where cross and joint recurrence plots are shown for CNST_e and KAIK_e (with trend). The same features are displayed as in the plots for the signal with no trend. The cross recurrence plot (Fig. 23a) shows attenuation of the cross recurrences away from the horizontal axis (as in Fig. 20c) whereas the joint recurrence plot (Fig. 23b) is essentially the same as Fig. 21f.

\section{Discussion}

Linear time series analysis concerns the manipulation of time series in order to characterise the statistics of the signal (mean, standard deviation, Fourier components of power spectrum) and/or remove noise (make the signal smoother, remove inliers) and/or make predictions or forecasts. Usually, techniques such as the Kalman filter or other forms of sequential Bayesian filters are employed (Jazwinski 1970; Young 2011). These methods do not always assume Gaussian distributions for the original data

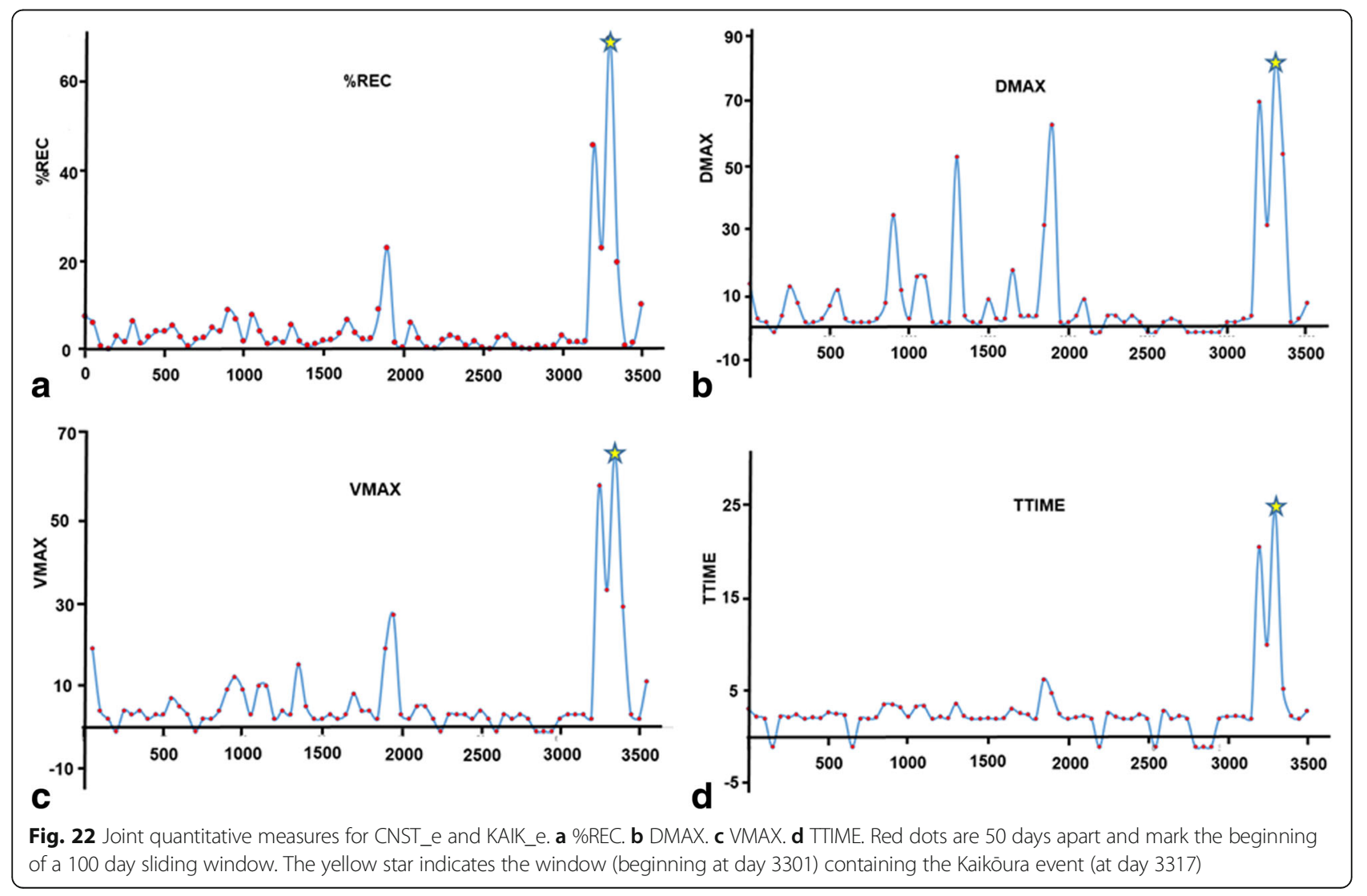



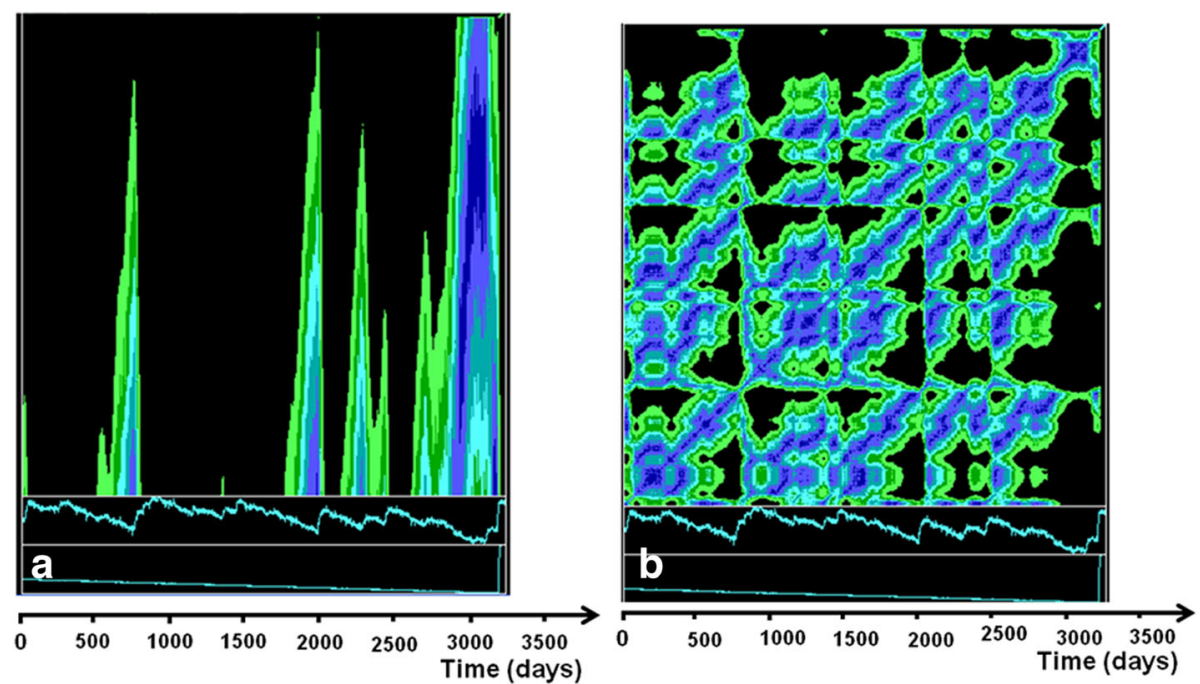

Fig. 23 Cross recurrence plot (a) and joint recurrence plot (b) for the raw CNST_e signal and the KAIK_e signal with the linear trend included. These plots are to be compared with Fig. 21e, $f$

and associated noise but commonly work best when the underlying statistics are Gaussian and the system is linear.

Nonlinear time series analysis, in contrast, is concerned with defining the dynamics of the processes that produced the signal rather than emphasising the statistics of the data. The dynamics are embodied in the attractor for the system which is the manifold that defines all possible states the system can occupy as it evolves no matter what the initial conditions are. The precise form of the attractor may be difficult to discern especially if the embedding dimension is large or has many "outliers" but some indications and/or constraints on its nature can be investigated using the ways in which the correlation dimension and false nearest neighbours scale with the embedding dimension. The output from a nonlinear signal analysis comprises estimates of the dimensions of the space in which the attractor exists (the embedding dimension) and other invariants such as the Rényi generalised dimensions, the entropy and Lyapunov exponent. If the embedding dimensions are small and the attractor is not too complicated, in the sense that state space is not too heterogeneous with respect to the density of states, then existing methods of nonlinear analysis work quite well. For high dimensional systems and complicated attractors it may be difficult to reach precise conclusions unless the data set is large enough to have completely sampled the attractor. Many of the pitfalls and problems are discussed by Small (2005) and McSharry (2011).

In this paper, we have reviewed, in a condensed manner, many aspects of nonlinear time series analysis with a view to focussing on a specific application, namely, GNSS data from New Zealand. The motivation for such studies is to characterise the dynamics of the processes that underlie the GNSS time series so that we learn more about the mechanisms that drive plate tectonics. GNSS data from five stations on the North Island of New Zealand have been analysed using recurrence plots and recurrence quantification analysis (RQA). The results of this analysis are shown in Table 3. We have also compared signals from two North Island stations with a station on the South Island that recorded displacements associated with the November 2016, magnitude 7.8 Kaikoura earthquake (Wallace et al. 2017) using cross and joint recurrence analysis.

For the five stations on the North Island, the embedding dimension (estimated as the dimension where the correlation dimension reaches a plateau when plotted against the embedding dimension) is approximately 10 . This value is comparable to that of many biological systems (Webber and Zbilut 2005). Although the embedding dimension is commonly inflated over the attractor dimension because of noise and non-stationary effects, this constrains the number of variables involved in the processes responsible for the underlying dynamics to $\leq$ 10. We consider such processes later in the "Discussion" section. The high dimensions of the attractor and its complexity are indicated by a delay construction of the attractor in three dimensions (Fig. 14). The attractor seems to have a number of knot-like outliers that may be visited only rarely so that it is necessary to have a very long time series to ensure all parts of the attractor have been sampled. The high dimensions of the attractor are confirmed by nonlinear prediction that gives reasonable results only if an embedding dimension of about 15 is used. 
A second invariant of importance is the first positive Lyapunov exponent which is a measure of how fast adjacent trajectories diverge as the system evolves; the larger this (positive) Lyapunov exponent the more chaotic the system (that is, the faster adjacent trajectories diverge). The RQA measure, DMAX, is inversely proportional to the largest positive Lyapunov exponent (Eckmann et al. 1987; Trulla et al. 1996) and so Table 2 shows that the signals from all five stations are chaotic from this point of view.

The entropy is the third invariant of importance and is a measure of the amount of information in the signal or of signal complexity or of how rapidly the information encoded in the current state of the system becomes irrelevant. The range for ENT in Table 2 is $4-5$ bits/bin. By comparison, the entropy for the classical Hénon attractor (Sprott 2003) is 2.557 bits/bin. These invariants enable us to define a chaotic system (or at least the chaotic systems we are interested in as geoscientists) as a bounded (does not grow without limits) system with deterministic dynamics (defined by an underlying set of physical and chemical processes) with a positive first Lyapunov exponent (neighbouring trajectories diverge exponentially with time).

The RQA measures for the five stations confirm that these data sets arise from chaotic dynamics. Generally the determinism is high and the systems are characterised by a large number of chaotic transitions (characterised by high percentage laminarity) which correspond to jumps in displacement at all scales.

\section{Synchronisation}

In most nonlinear systems, where sub-systems exist that are coupled together by the transfer of energy or mass, some form of synchronisation develops if the coupling strength is large enough (Romano Blasco 2004; Marwan et al. 2007a). Synchronisation in seismic systems has been discussed by Perez et al. (1996), Sammis and Smith (2013), Scholz (2010) and Bendick and Bilham (2017). Synchronisation develops because the coupling inhibits some frequencies and enhances others so that a particular range of frequencies survives in the system as a whole. Phase and/or frequency synchronisation are the commonly envisaged forms of synchronisation but these are actually relatively rare and the more common form is generalised synchronisation where the behaviour of one sub-system is a (generally nonlinear) function of the behaviour of other sub-systems. Generalised synchronisation is difficult to detect but cross and joint recurrence plots offer fast and efficient means of detection and investigation. In cross recurrent plots lines of synchronisation (LOSs) develop and the slope of these lines is $\tan ^{-1}\left(T_{1} / T_{2}\right)$ where $T_{1}$ and $T_{2}$ are the characteristic times of processes operating in two synchronised sub-systems.

Analysis (Fig. 18) shows that generalised synchronisation exists between CNST_e and PAWA_e signals in the North Island of New Zealand. The value of $\left(T_{\mathrm{CNST}} /\right.$ $\left.T_{\mathrm{PAWA}}\right)$ varies with time for these two sub-systems but averages about 3:5 (Fig. 18a). The synchronisation is well developed throughout the recorded displacement histories of the two stations. There are large gaps in synchronisation before PAWA events. Similar less defined gaps exist before major events for CNST_e. These gaps act as precursors to the main displacement events.

Generalised synchronisation also occurs between CNST and PAWA stations on the North Island and KAIK station on the South Island. For PAWA_e and KAIK_e synchronisation, a single LOS is developed with the ratio, $T_{\mathrm{PAWA}} / T_{\mathrm{KAIK}}$, varying from $1: 1$ to $>20: 1$ over the time records that are available. For CNST_e/KAIK_e synchronisation, the situation is a little more complicated. The ratio, $T_{\mathrm{CNST}} / T_{\mathrm{KAIK}}$, remains large $(\geq 20)$ throughout the recorded history of displacements but synchronisation occurs in bursts with each burst beginning about 100 days before a major displacement event at CNST and ending as the event begins. The Kaikoura event is preceded by 9 months of intense synchronisation and the synchronisation ceases as the Kaikoura event occurs.

The synchronisation between CNST and KAIK is made even more evident by a joint recurrence plot (Fig. 21f) and joint RQA (Fig. 22) where the system shows clear evidence of synchronisation beginning at least 100 days before the Kaikōura event.

\section{Precursors, predictions and forecasting}

Nonlinear forecasting or prediction, particularly with respect to process control, is now widely used in industrial applications ranging from control of lathe tool chatter (Abarbanel 1996), brake squeal (Oberst and Lai 2015) and chemical reactions (Petrov and Showalter 1997) to weather forecasting (Yoden 2007). With the development of new forecasting procedures such as shadowing algorithms (Stemler and Judd 2009), one can only expect forecasting to improve. The accuracy of such methods depends on how well the observed time series for the system has sampled the underlying attractor that describes the dynamics of that particular system. For attractors that are characterised by large embedding dimensions or that are topologically complicated, forecasting may be difficult. Moreover, we do not yet know if the attractors for GNSS systems evolve with time. There is a fundamental restriction that the trajectory of any chaotic system will diverge exponentially with time from any observed trajectory. The rate of divergence is measured by the magnitudes of the (positive) Lyapunov 
exponents. Thus, there is always an "horizon of predictability" for any chaotic system. At present, we do not know how far this "horizon" extends for GNSS time series but the single result reported in this paper shows promise; much more work is needed.

Despite the limitations mentioned above, the time histories of RQA measures for individual stations show that some act as precursors for main displacement events up to 100 days before an event. These results need to be studied and confirmed using time series with greater time resolution. Since data is collected at $1 \mathrm{~s}$ intervals a reasonable refinement of the data would to be to bin results at 0.1 day intervals rather than the present 1 day binning procedure. In doing so, it is important to pay attention to the binning procedure and ensure that dynamical noise is retained during the aggregation procedure. The definition of RQA for single stations that precede a major displacement event by considerable time periods is clearly an important goal for nonlinear time series analysis.

With respect to the overall strategy for forecasting of GNSS time series, the application of nonlinear analysis to data from one station can only aim to forecast future events for that particular station. A far more promising approach, motivated by the cross and joint recurrence results for CNST_e and KAIK_e and for PAWa_e and KAIK_e is to monitor cross and joint recurrence histories for a network of stations. This regional network approach has the promise to track the spatial evolution of synchronicity across the network and detect sites where abnormal synchronicity is evolving. The results of this paper (Figs. 21 and 22) show the intensity of synchronicity growing dramatically (such that the intensity of previous synchronous recurrences in the network are relatively small) for approximately 9 months before the Kaikoura event. The precise time of the Kaikoura event cannot be defined by the analysis but the risk could be quantified well ahead of the event. An extension of this cross/joint recurrence study to the whole of the GNSS network in New Zealand, so that patterns of synchronisation developed in historical time series over the past decade could be understood, would be a fundamental next step.

The advantage of a networked cross and joint recurrence system of monitoring is that the site of an imminent event can be pinpointed whereas the use of only two stations results in ambiguity regarding which of the two stations will host the event. Ambiguity can be further reduced if reliable RQA precursors for events at individual stations by sliding window analysis are integrated with results from synchronisation in the GNSS network.

\section{Underlying processes}

The processes that have been proposed to produce the spectrum of behaviours observed in the New Zealand tectonic system ranging from slow continuous displacement histories with small but distinct variability (PAWA prior to the 14 November, 2016, M7.8 Kaikoura earthquake), to repeated slow discontinuous events (CNST over a 10 year period), to major seismic events (the M7.8 Kaikoura earthquake) have been discussed by many authors (see papers referred to in Wallace et al. 2017). We break these processes into the following high level categories.

Deformation processes involving elastic-brittle-viscous behaviour of rocks in the upper crust with fluids present. The brittle behaviour seems to be associated with rate dependent weakening of the constitutive parameters (commonly expressed as velocity dependent frictional state variables) and some form of chemical healing of damage that has resulted from the brittle behaviour. The deformation process is exothermic (in the extreme case producing pseudotachylite melts) and involves the generation of dilatancy. The heat generated during deformation together with dilatancy influences the fluid pore pressure in the deforming rock mass.

Chemical processes involving the devolatilisation of hydrous and carbonate minerals including calcite, clays, micas and, in particular, serpentinite. These reactions are commonly endothermic and involve a positive $\Delta \mathrm{V}$ of reaction. Another chemical process is melting to produce pseudotachylites, also an endothermic process. Such processes compete in the heat budget with deformation. Another fundamental chemical process is the healing of damage which may involve hydrolysis and hence is exothermic and competes with the devolatilisation and melting processes for heat.

Hydraulic processes involving fluid flow within the deforming rock mass and especially away from chemical devolatilisation sites.

Thermal processes involving heat transport by both conduction and advection of heat in moving fluids. Heat is generated during deformation and hydrolysing reactions and removed from a deforming site by simultaneous endothermic reactions and heat transport.

These deformation-chemical-hydraulic-thermal processes are strongly coupled through the heat and mass budgets so that the behaviour of the system is nonlinear and expected to be chaotic. In such systems, it is common to explore any complexity that might arise in terms of dimensionless groups which parameterise the processes that occur and define the state hyperspace within which the system evolves (Aris 1999). In this case, we can identify the following dimensionless groups:

- The Damköhler number, $D a$, that is a measure of the importance of the time scale of fluid flow relative to that for chemical reaction. In many systems with coupled chemical reactions and fluid/ 
heat transport changes in $D a$ result in episodic behaviour of the chemical reaction and temperature (Gray and Scott 1990; Aris 1999).

- The Newtonian cooling time that measures the importance of the thermal cooling time scale relative to the chemical time scale. This is also important in controlling chemical reaction rates (Aris 1999). It is influenced strongly by the shape of the system and by the thermal conductivity of the wall rocks and so is important in studies of specific tectonic systems; we expect systems with different geometries to behave in different manners.

- The Arrhenius number, $A r$, that measures the importance of the thermal energy relative to the activation energy for chemical reactions and for rate sensitive deformation processes. Ar defines the spectrum of chemical reactions that occur in a particular system (Law 2006, pp. 60-62) and hence is fundamental in defining the order in which chemical reactions occur in a particular system. $A r$ is also fundamental in defining the deformation behaviour of the system (Veveakis et al. 2010).

- The Gruntfest number, $G r$, that measures the importance of the heat generated by deformation to that absorbed or generated by chemical reactions. $G r$ is important in defining the magnitude of the temperature increase possible for coupled deformation/chemical reactions and hence influences the deformation rate in systems where rate dependent constitutive behaviour is included (Veveakis et al. 2010, 2014; Alevizos et al. 2014; Poulet et al. 2014).

- The Lewis number, Le, that is a measure of the importance of heat transport by diffusion to mass transport. Le is important in defining the magnitude of the pore pressure increase possible for a particular temperature increase as fluid diffuses from the site of the temperature increase (Veveakis et al. 2014, 2017; Alevizos et al. 2014; Poulet et al. 2014). In tectonic systems, the permeability of the wall rocks for the system controls whether pore pressure leaks away as devolatilisation proceeds and the temperature increases. If the wall rocks are relatively impermeable, the Lewis number is small and the pore pressure increases would be large thus promoting brittle failure.

- In addition, other evolutionary processes need to be parameterised including rates of damage generation and of chemical healing, the temperature dependence of deformation and chemical reaction rates, and the influence of temperature changes and dilatancy on fluid pressure.
We propose that these dimensionless groups and associated evolution equations define the state space for crustal systems such as that examined for New Zealand so that the dimensions of the state space are relatively large as indicated by the recurrence analysis. Any computer models need to be constrained by the magnitude of the dimensions of state space indicated by the displacement histories. Some beginnings of coupled modelling are presented by Veveakis et al. (2010, 2014); Alevizos et al. (2014); Poulet et al. (2014) where close to periodic behaviour is modelled and such studies need to be extended to include the complete parameter space.

\section{Future directions}

This paper has highlighted the need for future work to be directed towards four objectives:

- Better definition of the attractor for the system.

- Better definition of precursors to major discontinuities in the displacement history.

- Better definition and understanding of the synchronisation system and dynamics of synchronisation, particularly the distribution of coupling strength between sites.

- Multi-dimensional recurrence network analysis aimed at establishing the underlying dynamics for the regional system.

These objectives can be addressed by a combination of longer time series (in order to ensure all parts of the attractor are sampled by the data) and better resolution within existing data sets in order to better resolve precursors and synchronisation frequencies. The former clearly will occur with continued data collection and the second requires binning the data in sizes smaller than 1 day (say 0.1 day intervals). Binning to small sizes requires attention to the process of aggregating data from the $1 \mathrm{~s}$ collection frequency to the 0.1 day time scale so that dynamical noise is preserved whilst observational noise is discarded.

Understanding the synchronisation dynamics requires nonlinear analysis of the complete networked New Zealand GeoNet GNSS measurement array. The synthesis of such a regional analysis might best be undertaken with some form of fusion between the concepts of recurrence networks (Small et al. 2009; Donner et al. 2010, 2011; Donges et al. 2012, 2015) and spatial network theory (Barthélemy 2011). This means that the regional GNSS array is to be regarded as a nonlinear dynamical network in which each node is coupled to every other node with variable coupling strengths between nodes. At each node, there is a nonlinear time series of displacements. Such a synthesis would better define the attractor for the integrated system as well as providing an understanding of 
the detailed dynamics of the system and of how the dynamics leads to synchronisation. An undertaking such as this is a major software development project and parts are being addressed at present. Some important developments for seismic spatial patterns are in Banish and Conrad (2014).

We conclude by speculating on a tectonic model that duplicates the synchronisation patterns revealed by the recurrence analysis in this paper. We propose, following Ben Zion (2008), that the crust of the Earth in the New Zealand region is close to a phase transition but is dominantly sub-critical so that there are no long-range correlations with respect to distortion of the plate. The plate as a whole continuously undergoes rigid body translations driven by plate tectonics and revealed by the calculations of Wallace et al. (2004). Internally, the plate undergoes a heterogeneous deformation with local shear strain rates varying from zero to $\approx 10^{-14} \mathrm{~s}^{-1}$ in places along the Great Alpine Fault (Beavan and Haines 2001). The plate is composed of microplates that undergo a spatially coordinated pattern of rotation, translation and internal deformation that maintains compatibility of the deformation gradient tensor.

The heterogeneous velocity field imposed on the crust by plate tectonic motions drives parts of the plate towards critical behaviour and this is expressed as increasing length scales for the correlation of distortions together with increasing length scales of synchronisation across the plate until criticality is reached at one or more places in the plate. The synchronisation stops and the plate becomes subcritical; the process then repeats itself. A test of such a model would be to show that some RQA measures scale as a power law with time before a large event as is the case for many systems as criticality is approached (Sethna 2011). Unfortunately, the data used here is not sufficiently closely spaced as to give the resolution for such a test. Perhaps, sampling at 0.1 day intervals would be sufficient to carry out such a study.

Finally, prediction within the system may be significantly enhanced by the use of new forecasting procedures such as the use of shadowing algorithms (Stemler and Judd 2009) coupled with ensemble modelling (Yoden 2007). In addition, other data sets, in particular, seismic data sets, need to be integrated with the GNSS data sets using cross- and joint-recurrence procedures.

\section{Conclusions}

The fundamental aim of the nonlinear analysis of time series is to reveal the dynamics of the processes that produced the signal. We have briefly reviewed the procedures involved in the nonlinear analysis of time series with particular emphasis on the notion of recurrence in nonlinear dynamical systems and indicated its application to some examples of GNSS time series from New Zealand sampled at 1 day intervals over $\approx 10$ years. This form of analysis is a powerful means of quantifying nonlinear aspects of the data, in discovering relationships that are not obvious in the raw data, in establishing precursors to singular events such as rapid jumps in displacements and for exploring synchronicity between displacement histories at adjacent and distant sites. The overall conclusions regarding the GNSS data sets examined are:

- The data are chaotic in the sense that they express the behaviour of a large bounded system, with a positive first Lyapunov exponent and result from deterministic dynamics.

- The embedding dimension for the attractor for this system is relatively large $(\approx 10)$ which is comparable with many biological systems and is to be expected for a system where coupled deformation-chemicalhydraulic-thermal processes are responsible for the dynamics.

- The entropy of these systems is moderate and comparable with that of many classical, relatively simple systems such as the Lorentz attractor. This means that predictive procedures have considerable potential.

- Precursors to strong discontinuities in the signal exist and in some cases precede the event by 100 days. Finer time resolution in the data sets (say binning at 0.1 days) would refine the precursors.

- Synchronisation between stations is common even over distances of $600 \mathrm{~km}$. The synchronisation is generalised synchronisation and the analysis reveals details of how the absolute and relative frequencies of recurrence vary both spatially and in time during synchronisation.

- Synchronisation between CNST and KAIK begins approximately 9 months before the Kaikoura magnitude 7.8 event. Such synchronisation (as revealed by cross-recurrence plots) provides a powerful means of forecasting this major seismic event and should be explored for other events.

- The displacement signals from the regional array are the response of a networked dynamical system where each node interacts with every other node with variable and evolving relations between the strength of coupling between nodes. Thus the behaviour of a specific site can only be understood and forecasted if the evolution of the whole system is monitored.

This paper shows that there is enough information in this regional nonlinear synchronised network to 
extract details of the underlying dynamics of the crustal deformation system and to provide precursors for major displacement events many months prior to an event. Investigations involving higher time-resolution and spatial recurrence network analysis should be pursued with vigour.

\section{Acknowledgements}

We thank Margaret Boettcher, Virginia Toy, Mark Munro and Klaus Gessner for the discussions that lead to this paper. Sebastian Orbest and Robert Niven are thanked for introducing us to recurrence analysis. We thank two anonymous reviewers for constructive comments that greatly improved the paper

\section{Funding}

No funding from an external source was involved in this study.

\section{Availability of data and materials}

All raw GNSS data used in this paper can be downloaded from http:// fits.geonet.org.nz/api-docs/

\section{Authors' contributions}

The topic was conceived jointly between BEH and AO. The first draft was written by $\mathrm{BEH}$ and subsequently added to and edited by $\mathrm{AO}$ who contributed substantially to the data processing. Both authors read and approved the final manuscript.

\section{Ethics approval and consent to participate}

Not applicable.

\section{Consent for publication}

Not applicable.

\section{Competing interests}

The authors declare that they have no competing interests.

\section{Publisher's Note}

Springer Nature remains neutral with regard to jurisdictional claims in published maps and institutional affiliations.

\section{Author details}

${ }^{1}$ Centre for Exploration Targeting, School of Earth Sciences, University of Western Australia, Perth, Western Australia 6009, Australia. ${ }^{2}$ School of Resources and Environmental Engineering, Hefei University of Technology, Hefei 230009, China. ${ }^{3}$ CSIRO, Perth, Western Australia 6102, Australia.

Received: 13 December 2017 Accepted: 25 June 2018

Published online: 23 July 2018

\section{References}

Abarbanel HDI (1996) Analysis of observed chaotic data. Springer, New York Aks DJ (2011) Analysis of recurrence: overview and application to eye-movement behaviour. Chapter 10. In: Nonlinear dynamical systems analysis for the behavioral sciences using real data. CRC Press, Boca Raton, pp 231-250

Alevizos S, Veveakis E, Poulet T (2014) Thermo-poro-mechanics of chemically active creeping faults: 1. Steady state. J Geophys Res Solid Earth 119:45584582

Aris R (1999) On some dynamical diagrams of chemical reaction engineering. Chaos 9:3-12

Arneodo A, Bacry E, Muzy JF (1995) The thermodynamics of fractals revisited. Physica A 213:232-275

Banish R, Conrad ND (2014) Cycle-flow-based module detection in directed recurrence networks EPL, 108: 68008-1-6

Barthélemy M (2011) Spatial networks. Phys Rep 499:1-101

Beavan J, Haines J (2001) Contemporary horizontal velocity and strain rate fields of the Pacific-Australian plate boundary zone through New Zealand. J Geophys Res 106:741-770

Beavan J, Matheson D, Denys P, Denham M, Herring T, Hager B, Molnar P (2004) A vertical deformation profile across the Southern Alps, New Zealand, from
3.5 years of continuous GPS data. In: van Dam T (ed) Cahiers de Centre Européen de Géodynamique et Séismologie, proceedings of the workshop: the state of GPS vertical positioning precision: separation of earth processes by space geodesy, vol 24

Beck C, Schlögl F (1995) Thermodynamics of chaotic systems. Cambridge University Press, New York

Ben Zion Y (2008) Collective behaviour of earthquakes and faults: continuumdiscrete transitions, progressive evolutionary changes and different dynamic regimes. Rev Geophys 46:RG4006

Bendick R, Bilham R (2017) Do weak global stresses synchronize earthquakes? Geophys Res Lett 44:8320-8327

Box GEP, Jenkins GM, Reinsel GC (2008) Time series analysis: forecasting and control. John Wiley \& Sons, Inc., Hoboken, New Jersey, p 746

Bröcker J, Parlitz U, Ogorzalek M (2002) Nonlinear noise reduction. Proc IEEE 90 898-918

Casdagli M (1997) Recurrence plots revisited. Physica D 108:12-44

Casdagli M, Eubank S (eds) (1992) Nonlinear modeling and forecasting. Westview Press, Redwood City, p 560

Chelidze T, Matcharashvili T (2015) Dynamical patterns in seismology. Chapter 10 In:Webber CL Jr., Marwan, N. Recurrence quantification analysis. Theory and best practices. Springer, Switzerland, pp 291-334.

Chen Q, Freymueller JT, Wang Q, Yang Z, Xu C, Liu J (2004) A deforming block model for present-day tectonics of Tibet. J Geophys Res 109:B01403

Crutchfield J (1979) Prediction and stability in classical mechanics. Seniour undergarduate thesis. University of California, Santa Cruz

Davies M (1993) Noise reduction by gradient descent. Int J Bifurcation Chaos 3: $113-118$

Donges JF, Heitzig J, Beronov B, Wiedermann M, Runge J, Feng QY, Tupikina L, Stolbova V, Donner RV, Marwan N, Dijkstra HA, Kurths J (2015) Unified functional network and nonlinear time analysis for complex systems science: the pyyunicorn package. Chaos 25:113101-1-25

Donges JF, Heitzig J, Donner RV, Kurths J (2012) Analytical framework for recurrence network analysis of time series. Phys Rev E 25:046105-1-16

Donner R, Small M, Donges N, Marwan N, Zou Y, Kurths J (2011) Recurrence based time series analysis by means of complex network methods. Int J Bifurcation Chaos 21:1019-1046

Donner RV, Zou Y, Donges JF, Marwan N, Kurths J (2010) Recurrence networks-a novel paradigm for nonlinear time series analysis. New J Physics 12:033025-1-40

Eckmann J-P, Komphorst SO, Ruelle D (1987) Recurrence plots of dynamical systems. Europhys Lett 5:973-977

Fan J, Gijbels I (1996) Local polynomial modelling and its applications: monographs on statistics and applied probability. Chapman \& Hall, London, p 341

Garcia SR, Romo MP, Figueroa-Nazuno J (2013) Characterization of ground motions using recurrence plots. Geofis Int 52:209-227

Goudarzi, M. A, Cocard, M, Santerre, R, Woldai T (2012). GPS interactive time series analysis software. GPS Solutions,

Grassberger PR, Hegger H, Kantz C, Schaffrath C, Schreiber T (1993) On noise reduction methods for chaotic data. Chaos 3:127-141

Gray P, Scott SK (1990). Chemical Oscillations and Instabilities. Oxford University Press, Oxford

Hobbs BE, Ord A (2015) Structural geology. Elsevier, Amsterdam

Hofmann-Wellenhof B, Lichtenegger H, Wasle E (2008) GNSS—global navigation satellite systems. Springer-Verlag, New York, p 516

Jazwinski AH (1970) Stochastic processes and filtering theory. Acad. Press, New York

Judd K (2003) Nonlinear state estimation, indistinguishable states, and the Kalman filter. Physica D 183:273-281

Judd K (2008a) Forecasting with imperfect models, dynamically constrained inverse problems, and gradient descent algorithms. Physica D 287:216-232

Judd K (2008b) Shadowing pseudo-orbits and gradient descent noise reduction. J Nonlinear Sci 18:57-74

Judd K, Stemler T (2009) Failure of sequential Bayesian filters and the success of shadowing filters in tracking nonlinear deterministic and stochastic systems. Phys Rev E 79:066206

Judd K, Stemler T (2010) Forecasting: it is not about statistics, it is about dynamics. Phil Trans R Soc A 3268:263-271

Kantz H (1994) Noise reduction by local reconstruction of the dynamics. In: Weigend AS, Gershenfeld NA (eds) Time series prediction: forecasting the future and understanding the past. SFI Studies in the Sciences of Complexity Proc. Vol. XV, Addison-Wesley, Reading, Massachusetts, pp 475-490 
Kantz H, Schreiber T (2004) Nonlinear time series analysis. Cambridge University Press, Cambridge

Law CK (2006) Combustion physics. Cambridge University Press, Cambridge

Lin M, Fan XX, Wang G, Zhao G (2016) Network structure entropy and its dynamical evolution for recurrence networks from earthquake magnitude time series. Eur Phys J B 89:131

Marwan N (2011) How to avoid potential pitfalls in recurrence plot based data analysis. Int. J. Bifurcation Chaos 21:1003-1017

Marwan N, Kurths J, Saparin P (2007b) Generalised recurrence plot analysis for spatial data. Phys Lett A 360:545-551

Marwan N, Romano MC, Thiel M, Kirths J (2007a) Recurrence plots for the analysis of complex systems. Phys Rep 438:237-329

McCaffrey R (2002) Crustal block rotations and plate coupling. In: Stein S, Freymueller J (eds) Plate boundary zones. Geodynamic series 30, vol 30. AGU, Washington, DC, pp 100-122

McCullough M, Sakellariuo K, Stemler T, Small M (2017) Regenerating time series from ordinal networks. Chaos 27:035814

McSharry PE (2011) The danger of wishing for chaos. Chapter 23. In: Guastello SJ (ed) Nonlinear dynamical systems analysis for the behavioral sciences using real data, CRC Press, Taylor \& Francis Group, Boca Raton

Moss F, McClintock PVE (1989) Noise in nonlinear dynamical systems. Cambridge University Press, Cambridge

Oberst S, Lai JCS (2015) Pad-mode-induced instantaneous mode instability for simple models of brake systems. Mech Syst Signal Process 62-63: 490-505

Ord A (1994) The fractal geometry of patterned structures in numerical models for rock deformation. In: Krühl JH (ed) Fractals and dynamic Systems in Geoscience. Springer-Verlag, Berlin, pp 131-155

Ord A, Munro M, Hobbs B (2016) Hydrothermal mineralising systems as chemical reactors: Wavelet analysis, multifractals and correlations. Ore Geology Reviews 79:155-179.

Packard NH, Crutchfield JP, Farmer JD, Shaw RS (1980) Geometry from a time series. Phys Rev Lett 45:712-716

Perez CJ, Corral A, Diaz-Guilera A, Christebsen K, Arenas A (1996) On selforganized criticality and synchronization in lattice models of coupled dynamical systems. Int J Mod Phys B 10, 1111-1151.

Petrov V, Showalter K (1997) Nonlinear prediction, filtering, and control of chemical systems from time series. Chaos 7:614-620

Poincaré H (1890) Sur la problem des trois corps et les équations de la dynamique. Acta Mathematica 13:1-271

Poulet T, Veveakis E, Regenauer-Lieb K, Yuen D (2014) Thermo-poro-mechanics of chemically active creeping faults: 3 . The role of serpentinite in episodic tremor and slip sequences, and transition to chaos. J Geophys Res Solid Earth 119:4606-4625

Romano Blasco MC (2004) Synchronisation analysis by means of recurrences in phase space. PhD thesis, University of Potsdam, p 108

Sammis C, Smith S (2013) Triggered tremor, phase-locking, and the global clustering of great earthquakes. Tectonophysics 589:167-171

Scholz CH (2010) Large earthquakes triggering, clustering and synchronization of faults. Bull Seismol Soc Amer 100:901-909

Sethna PJ (2011) Statistical mechanics: entropy, order parameters, and complexity. Clarendon Press, Oxford

Small M (2005) Applied nonlinear time series analysis applications in physics, physiology and finance. World Scientific, New Jersey, p 245

Small M, Zhang J, Xu X (2009) Transforming time series into complex networks. In: Zhou J (ed) Complex sciences. Complex 2009. Lecture notes of the Institute for Computer Sciences, Social Informatics and Telecommunications Engineering, vol 5. Springer, Berlin, pp 2078-2089

Sprott JC (2003) Chaos and time series analysis. Oxford University Press, Oxford, p 507

Stemler T, Judd K (2009) A guide to using shadowing filters for forecasting and state estimation. Physica D 238:1260-1273

Stoica P, Moss R (2005) Spectral analysis of signals. Prentice Hall, New Jersey

Takens F (1981) Detecting strange attractors in turbulence. In: Rand DA, Young LS (eds) Dynamical systems and turbulence. Warwick 1980 Proceedings, Lecture notes in mathematics, vol 898. Springer, New York, pp 366-381

Thatcher W (1995) Microplate versus continuum descriptions of active plate deformation. J Geophys Res 100:3885-3894

Thatcher W (2007) Microplate model for the present-day deformation of Tibet. J Geophys Res 112:B01401

Trulla LL, Giuliani A, Zbilut JP. Webber CL (1996) Recurrence quantification analysis of logistic equation with transients. Phys Lett A 223:255-260
Veveakis E, Alevizos S, Poulet T (2017) Episodic tremor and slip (ETS) as a chaotic multiphysics spring. Phys Earth Planet Inter 264, 20-34.

Veveakis E, Alevizos S, Vardoulakis I (2010) Chemical reaction capping of thermal instabilities during shear of frictional faults. J Mech Phys Solids 58:1175-1194

Veveakis E, Poulet T, Alevizos S (2014) Thermo-poro-mechanics of chemically active creeping faults: 2. Transient considerations. J Geophys Res Solid Earth 119:4583-4605

Wallace LM, Beavan J, McCaffrey R, Darby D (2004) Subduction zone coupling and tectonic block rotations in the North Island, New Zealand. J Geophys Res 109:B12406

Wallace LM, Ellis S, Mann P (2010) Tectonic block rotation, arc curvature, and back-arc rifting: insights into these processes in the Mediterranean and the western Pacific. In: 2008 IOP Conf. Ser., Earth Environ. Sci, vol 2, p 012010

Wallace LM, Kaneko Y, Hreinsdottir S, Mamling I, Peng Z, Bartlow N, D'Anasatasio FB (2017) Large-scale dynamic triggering of shallow slow slip enhanced by overlying sedimentary wedges. Nat Geosci 10:765-770

Webber CL, Zbilut JP (2005) Recurrence quantification analysis of nonlinear dynamical systems. Chapter 2. In: Riley MA, Van Orden G (eds) Tutorials in contemporary nonlinear methods for the behavioural sciences, pp 26-92 https://www.nsf.gov/pubs/2005/nsf05057/nmbs/nmbs.pdf. Accessed 5 July 2018

Weigend AS, Gershenfeld NA (eds) (1994) Time series prediction: forecasting the future and understanding the past. SFI Studies in the Sciences of Complexity, Proc. Vol. XV, Addison-Wesley, Reading, Massachusetts, 643 p

Yoden S (2007) Atmospheric predictability. J Met Soc Japan 85B:77-102

Young PC (2011) Recursive estimation and time-series analysis. Springer, Berlin

Zhang P-Z, Shen Z, Wang M et al (2004) Continuous deformation of the Tibetan Plateau from global positioning system data. Geology 32:809-812

\section{Submit your manuscript to a SpringerOpen ${ }^{\circ}$ journal and benefit from:}

- Convenient online submission

- Rigorous peer review

- Open access: articles freely available online

- High visibility within the field

- Retaining the copyright to your article

Submit your next manuscript at $>$ springeropen.com 